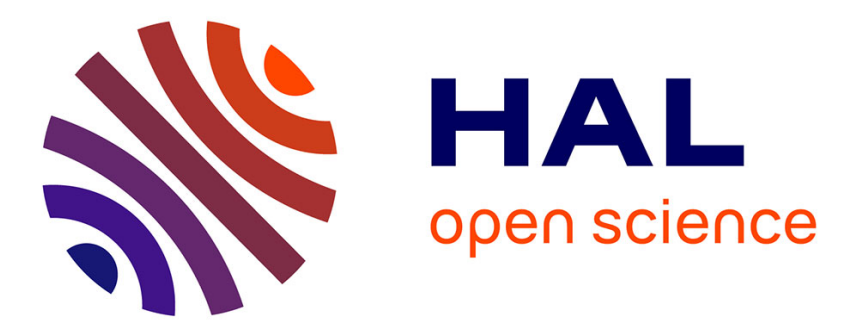

\title{
Matching of asymptotic expansions for wave propagation in media with thin slots. I. The asymptotic expansion
}

\author{
Patrick Joly, Sébastien Tordeux
}

\section{To cite this version:}

Patrick Joly, Sébastien Tordeux. Matching of asymptotic expansions for wave propagation in media with thin slots. I. The asymptotic expansion. Multiscale Modeling and Simulation: A SIAM Interdisciplinary Journal, 2006, 5 (1), pp.304-336 (electronic). 10.1137/05064494X . inria-00527588

\section{HAL Id: inria-00527588 \\ https://hal.inria.fr/inria-00527588}

Submitted on 13 Dec 2019

HAL is a multi-disciplinary open access archive for the deposit and dissemination of scientific research documents, whether they are published or not. The documents may come from teaching and research institutions in France or abroad, or from public or private research centers.
L'archive ouverte pluridisciplinaire HAL, est destinée au dépôt et à la diffusion de documents scientifiques de niveau recherche, publiés ou non, émanant des établissements d'enseignement et de recherche français ou étrangers, des laboratoires publics ou privés. 


\title{
MATCHING OF ASYMPTOTIC EXPANSIONS FOR WAVE PROPAGATION IN MEDIA WITH THIN SLOTS I: THE ASYMPTOTIC EXPANSION*
}

\author{
PATRICK JOLY ${ }^{\dagger}$ AND SÉBASTIEN TORDEUX ${ }^{\ddagger}$
}

\begin{abstract}
In this series of two articles, we consider the propagation of a time harmonic wave in a medium made of the junction of a half-space (containing possibly scatterers) with a thin slot. The Neumann boundary condition is considered along the boundary on the propagation domain, which authorizes the propagation of the wave inside the slot, even if the width of the slot is very small. We perform a complete asymptotic expansion of the solution of this problem with respect to the small parameter $\varepsilon / \lambda$, the ratio between the width of the slot, and the wavelength. We use the method of matched asymptopic expansions which allows us to describe the solution in terms of asymptotic series whose terms are characterized as the solutions of (coupled) boundary value problems posed in simple geometrical domains, independent of $\varepsilon / \lambda$ : the (perturbed) half-space, the half-line, a junction zone. In this first article, we derive and analyze, from the mathematical point of view, these boundary value problems. The second one will be devoted to establishing error estimates for truncated series.
\end{abstract}

Key words. slit, slot, wave equation, Helmholtz equation, approximate model, matching of asymptotic expansions

AMS subject classifications. 35J05, 34E05, 78A45, 78A50

DOI. $10.1137 / 05064494 \mathrm{X}$

1. Introduction. In many practical applications concerning time harmonic (namely the pulsation $\omega$ or the wavelength $\lambda$ is given) electromagnetic or acoustic wave propagation, the problems to be solved involve the presence of structures with at least one space dimension of characteristic length $\varepsilon \ll \lambda$. In this paper, we are considering the treatment of two-dimensional (2D) thin slots which corresponds typically to the geometry of Figure 1.1. For the applications, the physically interesting situation (for example the situation one meets with the microwave shielding of thin slots [3] or flanged waveguide antennas [20]; see [9] for more examples) corresponds to

$$
\lambda / 1000<\varepsilon<\lambda / 10, \quad \varepsilon<L / 10, \quad \text { and } \quad \lambda / 10<L<10 \lambda,
$$

where $\lambda$ is the wavelength, $\varepsilon$ is the width of the slot, and $L$ the length of the slot.

For numerical simulation of wave propagation in media with thin slots, a natural idea is to derive an approximate "1D-2D" model: a one-dimensional (1D) model, posed on the curve that materializes the limit of the slot when $\varepsilon$ tends to 0 , for the propagation inside the slot and a $2 \mathrm{D}$ model for the rest of the computational domain. The main difficulty consists in finding the technique (not unique) used for coupling the two models. Such models have been designed in the engineering literature (see [18], [19], [8], [9], or [10] for a review) and are commonly used in various computational codes. However, the complete understanding and evaluation of such models suffers, in our opinion, from a lack of mathematical analysis.

\footnotetext{
${ }^{\dagger}$ Projet POEMS, Batiment 13, INRIA, Domaine de Voluceau - Rocquencourt - B.P. 105, 78153 Le Chesnay Cedex, France (patrick.joly@inria.fr).

‡ Seminar for Applied Mathematics, ETH Zurich, Rämistrasse 101, 8092 Zurich, Switzerland (tordeux@math.ethz.ch).
} 


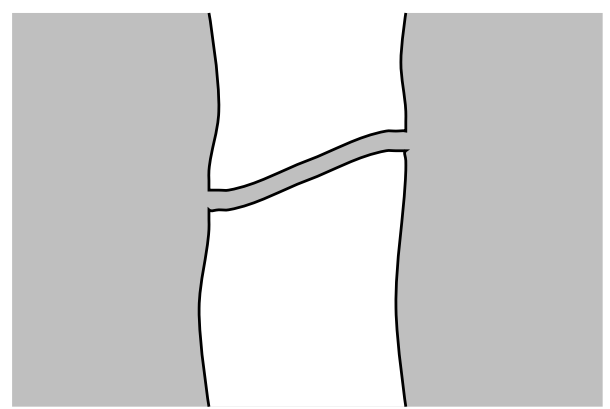

FIG. 1.1. Geometry of the domain of propagation.

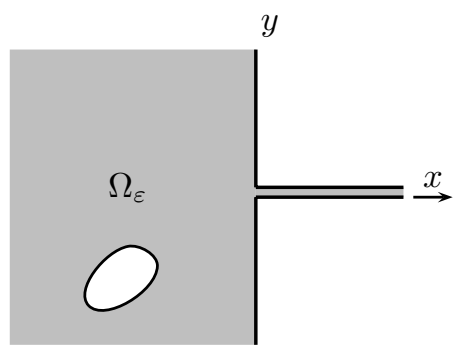

FIG. 1.2. Domain for the model problem.

In a previous paper [1], in the case of the scalar Helmholtz equations with homogeneous Neumann boundary conditions (the interesting case from the physical point of view) we proposed and analyzed in detail a 1D-2D model by a "brute matching" of the $1 \mathrm{D}$ and $2 \mathrm{D}$ models, in the spirit of what is done in the engineering literature. This work justifies completely this type of approach from the mathematical point of view (sharp error estimates are given) but also emphasizes its limitation: the accuracy is, roughly speaking, limited to $O\left(\varepsilon^{2}\right)$.

A natural idea is to try to develop more accurate approximate models. Of course, this requires a (more) complete description of the asymptotic behavior of the solution when $\varepsilon$ tends to 0 . This is precisely the objective of the present article, which is the first of a series of two papers devoted to the asymptotic analysis of the model problem of the semi-infinite straight slot. Typically, the domain of propagation is the union of a half-space (that may contain, for instance, an obstacle) and a thin semi-infinite straight slot, orthogonal to the half-space (see Figure 1.2):

$$
\bar{\Omega}_{\varepsilon}=\bar{\Omega}_{H} \cup \overline{\Omega_{S}^{\varepsilon}},
$$

with

$$
\begin{gathered}
\Omega_{S}^{\varepsilon}=\left\{(x, y) \in \mathbb{R}^{2} / 0<x \text { and }-\varepsilon / 2<y<\varepsilon / 2\right\}, \\
\Omega_{H}=\left\{\mathbf{x}=(x, y) \in \mathbb{R}^{2} / x<0 \text { and } \mathbf{x} \notin \mathcal{B}\right\},
\end{gathered}
$$

where $\mathcal{B}$ is a regular obstacle included in the left half-space $x<0$. The problem we consider is $^{1}$

\footnotetext{
${ }^{1}$ We have chosen to use the definition $u \in H_{l o c}^{1}(D) \Longleftrightarrow \varphi u \in H^{1}(D)$ for all $\varphi \in \mathcal{D}\left(\mathbb{R}^{2}\right)$.
} 


$$
\left\{\begin{array}{l}
\text { Find } u^{\varepsilon} \in H_{l o c}^{1}\left(\Omega_{\varepsilon}\right) \text { outgoing such that } \\
\Delta u^{\varepsilon}+\omega^{2} u^{\varepsilon}=-f \text { in } \Omega_{\varepsilon}, \\
\frac{\partial u^{\varepsilon}}{\partial n}=0 \text { on } \partial \Omega_{\varepsilon},
\end{array}\right.
$$

where $f \in L^{2}\left(\Omega_{H}\right)$ is the compactly supported data. Classically, by outgoing solution, we mean that we prescribe a given behavior at infinity to the solution; namely,

- at infinity in $\Omega_{H}, u^{\varepsilon}$ satisfies the Sommerfeld radiation condition [2];

- inside the slot the solution is the superposition of modes which are either evanescent or propagating in the direction $x>0$ (see [1]).

REMARK 1.1. The case of a slot of finite length is of the same type of analysis, modulo some additional technical difficulties, except, however, for some critical lengths $L$ linked to resonance phenomena (see [6]).

For studying the behavior of $u^{\varepsilon}$ for small $\varepsilon$, we will use the method of matched asymptotic expansions. This is a well-known method that has been developed in the beginning of the 1970's, initially to analyze boundary layer phenomena. This approach has been developed quasi-independently (one does not find a lot of cross citations in the publications) by two mathematical schools, from two rather different points of view:

- The British School's effort has been put on the machinery of formal asymptotics and matching principles (see [5] for the basic tools or [16] for a review).

- The Russian School has investigated more deeply the rigorous justification, far from being easy, of this type of formal expansion (see [4] for the justification of the spatial expansions and [17] for a review on the matching of asymptotic expansions).

In this work, we intend to apply this technique and analyze it rigorously for the model problem. This problem has already been studied via matched asymptotics in [21], where the authors restrict themselves to the first orders of the expansions, without paying attention to the mathematical justification.

REMARK 1.2. The fact that the method of matched asymptotic expansions is most often presented as a formal technique is a major drawback in the opinion of a lot of mathematicians who prefer the so-called multiscale technique, which appears to benefit from a rigorous framework (the reader can refer to $[15,14,13,11]$ to obtain explanations on the multiscale technique). With this work, we also want to convince the reader that the method of matched asymptotic expansions is not only a formal technique.

As it is classical with this type of problem, the work can be naturally divided into three steps. The first one is related to obtaining the formal asymptotic expansion and constitutes the most algebraic and calculatory part of the work. The next two cover two different aspects of the mathematical justification and make use of various techniques for the analysis of PDEs.

Step 1. Derive the formal expansion. One starts from an ansatz, i.e., an a priori form for the asymptotic expansion that is injected into the equations of the initial ( $\varepsilon$ dependent) problem, to deduce a series of ( $\varepsilon$ independent) elementary problems that are supposed to characterize the different terms of the asymptotic expansion.

Step 2. Show that the various terms of the asymptotic expansions are well defined, i.e., that the above elementary problems are well posed. This is not 
necessarily straightforward since these problems are quite often nonstandard. Step 3. Justify the asymptotic expansion, namely establish error estimates between the true solution and truncated asymptotic expansions.

For the clarity of the exposition, we have chosen to separate our presentation into two distinct articles. In the present one, the first part, we treat essentially Steps 1 and 2. We also state the main theorems (error estimates) whose proofs, postponed to the second article, will use in particular the stability and consistency arguments that have been developed in [1].

The outline of this paper is as follows.

In section 2, we will give the forms of the asymptotic expansions in the three different zones (far-field, near-field, and slot-field zones) and state our main results, namely Theorems 2.1, 2.2, and 2.3.

Section 3 is devoted to Step 1. First, we introduce some preliminary materials: asymptotic behavior of solutions of Helmholtz and Laplace equations. We then give the coupled problem (see section 3.3) which defines the terms of the asymptotic expansions. Finally, we present the computations which allow us to derive this problem.

In section 4, we prove that the coupled problem is well posed (this is Step 2). The existence proof is based on the introduction of special functions which are constructed in the appendix.

\section{The asymptotic expansions and associated estimates.}

2.1. The formal expansions. For this type of problem involving several space scales, it is not possible to write a uniform expansion for the solution everywhere in the domain $\Omega_{\varepsilon}$. Here we will have to consider three distinct zones, respectively the far-field zone, the near-field zone, and the slot zone, in which different expansions are obtained. The denominations "far" and "near" refer to the position with respect to the end point of the slot.

In the following, we will denote by $\mathcal{C}$ the class of positive continuous functions of $\varepsilon>0$ that tend to 0 when $\varepsilon \rightarrow 0$, less rapidly than linearly,

$$
\mathcal{C}=\left\{\eta: \mathbb{R}_{*}^{+} \rightarrow \mathbb{R}_{*}^{+} / \lim _{\varepsilon \rightarrow 0} \eta(\varepsilon)=0, \text { and } \lim _{\varepsilon \rightarrow 0} \eta(\varepsilon) / \varepsilon=+\infty\right\}
$$

and will introduce four functions $\eta_{H}^{ \pm}$and $\eta_{S}^{ \pm}$in $\mathcal{C}$ satisfying

$$
\eta_{H}^{-}(\varepsilon)<\eta_{H}^{+}(\varepsilon) \text { and } \eta_{S}^{-}(\varepsilon)<\eta_{S}^{+}(\varepsilon) .
$$

Because of the 2D context, polynomial logarithmic gauge functions will be used for describing the asymptotic behavior of $u^{\varepsilon}$, namely the functions

$$
\left(\frac{\omega \varepsilon}{2}\right)^{i} \log ^{k}\left(\frac{\omega \varepsilon}{2}\right)
$$

More precisely, we will use the set of indices (see Figure 2.1)

$$
\mathbf{J}=\{(i, k) \in \mathbb{N} \times \mathbb{N} / k \leqslant i\},
$$

which we shall furnish with the total order relation

$$
(j, l) \leqslant(i, k) \Longleftrightarrow \quad j<i \quad \text { or } \quad j=i \text { and } l \leqslant k
$$

illustrated by Figure 2.2. 


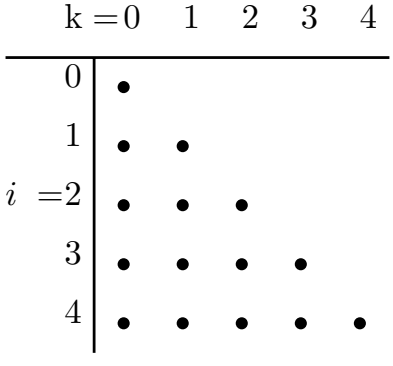

FIG. 2.1. The index set $\mathbf{J}$.

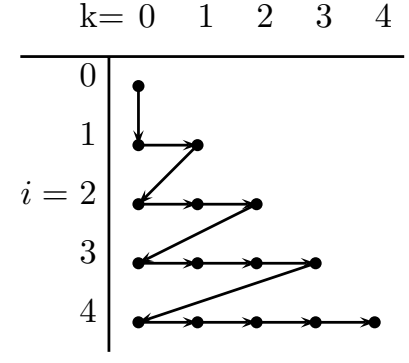

FIG. 2.2. The order relation in $\mathbf{J}$.

REMARK 2.1. Here we have used $\omega \varepsilon / 2$ for the small parameter. This is justified by the fact that $\omega \varepsilon$ has no dimension. The factor $1 / 2$ has simply a technical interest.

The far-field (half-space) expansion. Roughly speaking, the far-field domain is $\Omega_{H}$ (the half-space without the obstacle $\mathcal{B}$ ) in which the solution $u^{\varepsilon}$ is searched of the form

$$
u^{\varepsilon}=\sum_{i=0}^{+\infty} \sum_{k=0}^{i}\left(\frac{\omega \varepsilon}{2}\right)^{i} \log ^{k}\left(\frac{\omega \varepsilon}{2}\right) u_{i}^{k}+o\left(\varepsilon^{\infty}\right) \text { in } \Omega_{H} .
$$

This expansion will not be valid uniformly in $\Omega_{H}$ but only in a far-field zone of the form

$$
\Omega_{H}(\varepsilon)=\left\{\mathbf{x} \in \Omega_{H} /|\mathbf{x}-\mathbf{0}|>\eta_{H}^{-}(\varepsilon)\right\} .
$$

Note that the domain $\Omega_{H}(\varepsilon)$ converges to $\Omega_{H}$ (see Figure 2.3) as $\varepsilon$ tends to 0 .

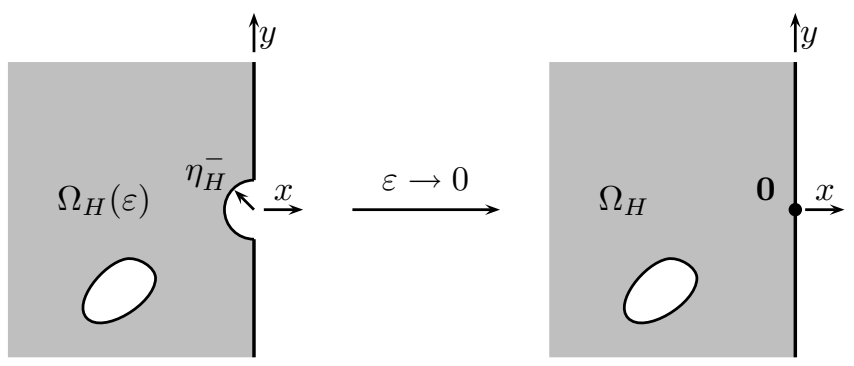

FIG. 2.3. The far-field domain.

The slot-field expansion. The slot-field domain is denoted by $\Omega_{S}^{\varepsilon}$; see Figure 2.4. In such a domain, it is natural to introduce the scaling $(x, Y)=(x, y / \varepsilon)$ in such a way that

$$
\left.(x, y) \in \Omega_{S}^{\varepsilon} \Longleftrightarrow(x, y / \varepsilon) \in \widehat{\Omega}_{S}=\right] 0,+\infty[\times]-1 / 2,1 / 2[,
$$

where $\widehat{\Omega}_{S}$ is a normalized (or canonical) slot of width 1 . In $\Omega_{S}^{\varepsilon}$, we shall look for $u^{\varepsilon}$ of the form

$$
u^{\varepsilon}(x, \varepsilon Y)=U^{\varepsilon}(x, Y)=\sum_{i=0}^{+\infty} \sum_{k=0}^{i}\left(\frac{\omega \varepsilon}{2}\right)^{i} \log ^{k}\left(\frac{\omega \varepsilon}{2}\right) U_{i}^{k}(x)+o\left(\varepsilon^{\infty}\right), \quad(x, Y) \in \Omega_{S},
$$




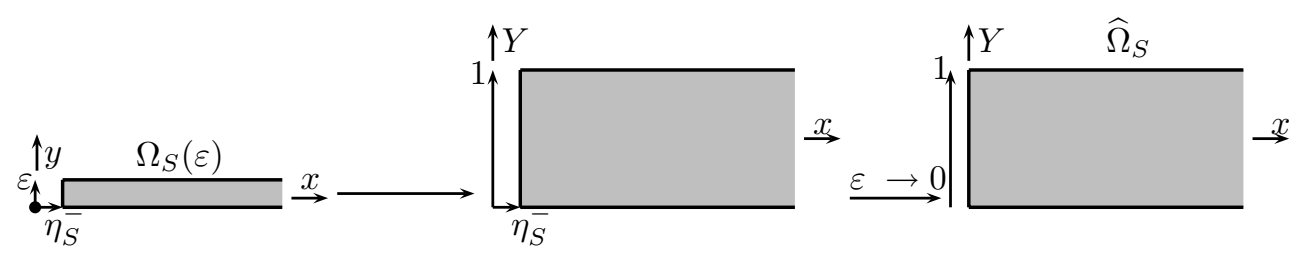

FiG. 2.4. Slot-field domain.

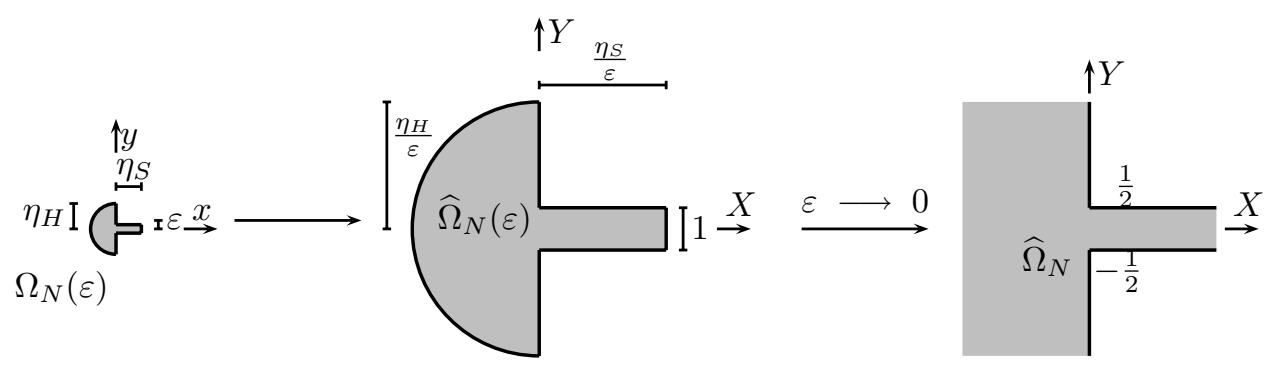

FIG. 2.5. Near-field domain.

where the functions $U_{i}^{k}$ 's are defined on $\mathbb{R}^{+}$.

REMARK 2.2. In fact, one does not need to assume a priori that $U_{i}^{k}$ does not depend on $Y$ : this follows from the calculations (see section 3.4). The $Y$-dependence of $U^{\varepsilon}$ is hidden in the $o\left(\varepsilon^{\infty}\right)$ term.

Once again, such an expansion will not be valid uniformly in $\widehat{\Omega}_{S}$ but only in a slot-field zone of the form

$$
\Omega_{S}(\varepsilon)=\left\{\mathbf{x}=(x, y) \in \Omega_{S}^{\varepsilon} / x>\eta_{S}^{-}(\varepsilon)\right\} .
$$

The near-field expansion. The near-field zone is defined by

$$
\overline{\Omega_{N}(\varepsilon)}=\left\{\mathbf{x} \in \overline{\Omega_{H}} /|\mathbf{x}| \leqslant \eta_{H}^{+}(\varepsilon)\right\} \cup\left\{(x, y) \in \overline{\Omega_{S}^{\varepsilon}} / x \leqslant \eta_{S}^{+}(\varepsilon)\right\} .
$$

In this zone we use the classical scaling $X=x / \varepsilon$ and $Y=y / \varepsilon$ in such a way that

$$
(x, y) \in \Omega_{N}(\varepsilon) \Longleftrightarrow(x / \varepsilon, y / \varepsilon) \in \widehat{\Omega}_{N}(\varepsilon),
$$

where, since $\eta_{H}^{+}$and $\eta_{S}^{+}$belong to $\mathcal{C}$, when $\varepsilon$ tends to $0, \Omega_{N}(\varepsilon)$ collapses to the origin and $\widehat{\Omega}_{N}(\varepsilon)$ converges to the canonical infinite open domain $\widehat{\Omega}_{N}$ (see Figure 2.5) defined by

$$
\widehat{\Omega}_{N}=(]-\infty, 0[\times \mathbb{R}) \cup\left(\left[0,+\infty[\times]-\frac{1}{2}, \frac{1}{2}[) .\right.\right.
$$

In $\Omega_{N}(\varepsilon), u^{\varepsilon}$ will be of the form

$$
\begin{array}{r}
u^{\varepsilon}(\varepsilon X, \varepsilon Y)=\mathcal{U}^{\varepsilon}(X, Y)=\sum_{i=0}^{+\infty} \sum_{k=0}^{i}\left(\frac{\omega \varepsilon}{2}\right)^{i} \log ^{k}\left(\frac{\omega \varepsilon}{2}\right) \mathcal{U}_{i}^{k}(X, Y)+o\left(\varepsilon^{\infty}\right), \\
(X, Y) \in \widehat{\Omega}_{N}(\varepsilon),
\end{array}
$$

where the $\mathcal{U}_{i}^{k}$ are complex-valued functions defined in $\widehat{\Omega}_{N}$. 
2.2. The main results. In this section, we state three theorems that specify the sense to be given to the expansions (2.6), (2.8), and (2.12). The proof of these theorems (a version of which is given in [12]) is postponed to the second article. Note also that these theorems refer to section 3 where the (coupled) problems defining the various functions appearing in these expansions are presented. Implicitly, it also refers to section 4 , where we shall prove that these problems are well posed.

THEOREM 2.1. There exists a unique family of functions $\left\{u_{i}^{k} \in C^{\infty}\left(\overline{\Omega_{H}} \backslash\{\mathbf{0}\}\right)\right.$, $(i, k) \in \mathbf{J}\}$, defined in section 3 (in particular $u_{k}^{k} \equiv 0$ for $k \neq 0$ ), such that for any compact set $F_{H} \subset \overline{\Omega_{H}} \backslash\{\mathbf{0}\}$ and any $N \in \mathbb{N}$, there exists $C_{N}\left(\omega, F_{H}, \operatorname{supp}(f)\right)>0$ such that

$$
\left\|u^{\varepsilon}-\sum_{i=0}^{N} \sum_{k=0}^{i}\left(\frac{\omega \varepsilon}{2}\right)^{i} \log ^{k}\left(\frac{\omega \varepsilon}{2}\right) u_{i}^{k}\right\|_{H^{1}\left(F_{H}\right)} \leqslant C_{N}\left(\frac{\omega \varepsilon}{2}\right)^{N+1}\left|\log \left(\frac{\omega \varepsilon}{2}\right)\right|^{N}\|f\|_{L^{2}} .
$$

The function $u_{0}^{0}$ is the natural limit of $u^{\varepsilon}$, i.e., the solution of the problem without a slot (see section 3.1).

Anticipating section 3.3, let us emphasize that the $u_{i}^{k}$ are not smooth up to the origin. For $i \neq 0$, each of the $u_{i}^{k}$ 's is singular near $\mathbf{0}$ so that $u_{i}^{k} \notin H_{l o c}^{1}\left(\Omega_{H}\right)$. More precisely, the singularity "increases" with $i-k$ in the sense that, when $r=|\mathbf{x}| \rightarrow 0$,

$$
u_{i}^{k}=O\left(r^{-i+k+1}\right) \quad \text { for } 0 \leqslant k \leqslant i-2 \quad \text { and } \quad u_{i}^{i-1}=O(\log r) .
$$

The convergence rate in the right-hand side of (2.13) is as expected: it corresponds to the order of magnitude of the first neglected term in the complete series expansion. On the other hand, we cannot claim that the series (2.6) converges, since the constant $C_{N}$ depends on $\mathrm{N}$.

Theorem 2.2. There exists a unique family $\left\{U_{i}^{k} \in C^{\infty}\left(\mathbb{R}^{+}\right),(i, k) \in \mathbf{J}\right\}$, defined in section 3 (in particular $U_{i}^{k}(x)=U_{i}^{k}(0) \exp \mathbf{i} \omega x$ ), such that for any compact set $F_{S} \subset\left\{(x, y) \in \widehat{\Omega}_{S} / x \neq 0\right\}$ and any $N \in \mathbb{N}$, there exists a positive constant $C_{N}\left(\omega, F_{S}, \operatorname{supp}(f)\right)$ such that

$\left\|U^{\varepsilon}-\sum_{i=0}^{N} \sum_{k=0}^{i}\left(\frac{\omega \varepsilon}{2}\right)^{i} \log ^{k}\left(\frac{\omega \varepsilon}{2}\right) U_{i}^{k}\right\|_{H^{1}\left(F_{S}\right)} \leqslant C_{N}\left(\frac{\omega \varepsilon}{2}\right)^{N+1}\left|\log \left(\frac{\omega \varepsilon}{2}\right)\right|^{N+1}\|f\|_{L^{2}}$.

THEOREM 2.3. There exists a unique family of functions $\left\{\mathcal{U}_{i}^{k} \in C^{\infty}\left(\widehat{\Omega}_{N}\right),(i, k) \in\right.$ $\mathbf{J}\}$, defined in section 3 , such that for any compact subset $F_{N}$ of ${\widehat{\Omega_{N}}}_{\text {and }}$ any $N \in \mathbb{N}$, there exists $C_{N}\left(\omega, F_{N}, \operatorname{supp}(f)\right)>0$ such that

$$
\left\|\mathcal{U}^{\varepsilon}-\sum_{i=0}^{N} \sum_{k=0}^{i}\left(\frac{\omega \varepsilon}{2}\right)^{i} \log ^{k}\left(\frac{\omega \varepsilon}{2}\right) \mathcal{U}_{i}^{k}\right\|_{H^{1}\left(F_{N}\right)} \leqslant C_{N}\left(\frac{\omega \varepsilon}{2}\right)^{N+1}\left|\log \left(\frac{\omega \varepsilon}{2}\right)\right|^{N+1}\|f\|_{L^{2}}
$$

Anticipating section 3 , let us mention that, except for $i=k$, the $\mathcal{U}_{i}^{k}$ are growing at infinity. More precisely,

$$
\left\{\begin{array}{l}
\mathcal{U}_{i}^{k}=O\left(\rho^{i-k}\right) \text { when } \rho=\left(X^{2}+Y^{2}\right)^{\frac{1}{2}} \rightarrow+\infty \text { in the half-space } X<0 \\
\mathcal{U}_{i}^{k}=O\left(X^{i-k}\right) \text { when } X \rightarrow+\infty \text { in the normalized slot } X>0
\end{array}\right.
$$


3. The coupled problems satisfied by the terms of the asymptotic expansions. In this section, we first derive the so-called limit fields corresponding to $(i, k)=(0,0)$ (section 3.1). Then we introduce in section 3.2 some preliminary notions that are needed for the description of the complete asymptotic expansion. In section 3.3 , we present a problem that will characterize the fields $u_{i}^{k}, \mathcal{U}_{i}^{k}, U_{i}^{k}$. The way this problem is obtained is given in section 3.4, where we derive the equations and boundary conditions satisfied by the fields $\left(u_{i}^{k}, \mathcal{U}_{i}^{k}, U_{i}^{k}\right)$ and explain how to obtain the matching conditions relating these fields.

3.1. The limit fields. As it is intuitively expected (and already proven in [1]), the field $u_{0}^{0}$, the limit of $u^{\varepsilon}$ when $\varepsilon \rightarrow 0$ in the domain $\Omega_{H}$, is nothing but the solution of the slotless problem posed in $\Omega_{H}$ :

$$
\left\{\begin{array}{l}
\text { Find } u_{0}^{0} \in H_{l o c}^{1}\left(\Omega_{H}\right) \text { outgoing such that } \\
-\Delta u_{0}^{0}-\omega^{2} u_{0}^{0}=f \text { in } \Omega_{H} \text { and } \frac{\partial u_{0}^{0}}{\partial n}=0 \text { on } \partial \Omega_{H} .
\end{array}\right.
$$

The other limit fields, namely the near-field $\mathcal{U}_{0}^{0}$ and the slot $U_{0}^{0}$, are also quite easy to guess:

- $\mathcal{U}_{0}^{0}$ is a constant in $\widehat{\Omega}_{N}$ equal to the value at the origin of the limit far-field $u_{0}^{0}$ :

$$
\mathcal{U}_{0}^{0}(X, Y)=u_{0}^{0}(\mathbf{0}) \text { for }(X, Y) \in \widehat{\Omega}_{N} .
$$

- $U_{0}^{0}$ is the $1 \mathrm{D}$-field, is independent of $Y$, and is the outgoing solution of the Helmholtz equation in the half-line $x>0$, whose value at $x=0$ coincides with the value at the origin of the limit far-field $u_{0}^{0}$, namely

$$
U_{0}^{0}(x, Y)=u_{0}^{0}(\mathbf{0}) \exp \mathbf{i} \omega x \quad \text { for }(x, Y) \in \widehat{\Omega}_{S} .
$$

3.2. Towards the construction of $\left(u_{i}^{k}, U_{i}^{k}, \mathcal{U}_{i}^{k}\right)$ : Preliminary material. This section is devoted to present useful results and notions about the solutions of homogeneous Hemholtz equations and "embedded" Laplace equations that naturally appear when applying the technique of matched asymptotic expansions. These results are needed to understand the coupled problem of section 3.3.

3.2.1. Bessel functions and related results. In what follows, we shall make extensive use of the Bessel functions $J_{p}(z)$ and $Y_{p}(z)$ for $p \in \mathbb{N}$ (see, for example, [22]). We shall use more particularly the generalized Taylor series expansion of these functions (one of their possible definitions)

$$
\left\{\begin{aligned}
J_{p}(z) & =\sum_{l=-\infty}^{+\infty} J_{p, l}\left(\frac{z}{2}\right)^{l} \\
Y_{p}(z) & =\sum_{l=-\infty}^{+\infty} Y_{p, l}\left(\frac{z}{2}\right)^{l}+\sum_{l=-\infty}^{+\infty} \frac{2}{\pi} J_{p, l}\left(\frac{z}{2}\right)^{l} \log \frac{z}{2}
\end{aligned}\right.
$$

where the numbers $J_{p, l}$ are given by

$$
\left\{\begin{array}{lll}
J_{p, p+l}=0 & \text { if } l<0 \text { or } l \text { odd } \\
J_{p, p+2 l}=\frac{(-1)^{l}}{l !(l+p) !} & \text { if } l \geq 0
\end{array}\right.
$$


and the numbers $Y_{p, l}$ are given by

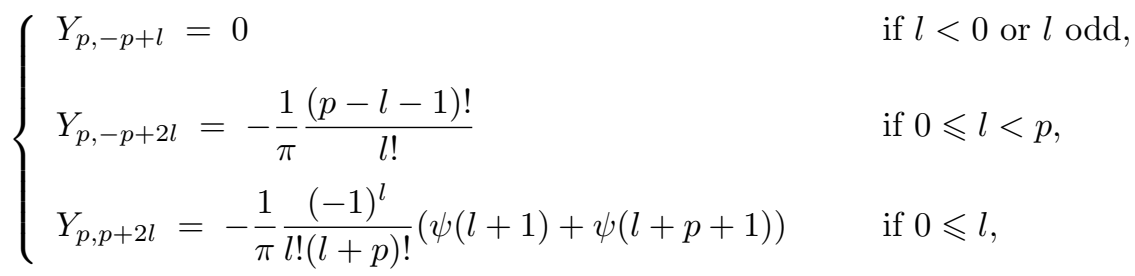

with

$$
\psi(1)=-\gamma, \quad \psi(k+1)=-\gamma+\sum_{m=1}^{k} \frac{1}{m} \quad \forall k \in \mathbb{N}^{*} .
$$

We emphasize here the fact that $J_{p}$ is regular at the neighborhood of $z=0$, while $Y_{p}$ is singular:

$$
J_{p}(z) \sim \frac{(z / 2)^{p}}{p !}, \quad Y_{0}(z) \sim \frac{2}{\pi} \log \left(\frac{z}{2}\right), \quad Y_{p}(z) \sim-\frac{(p-1) !}{\pi}\left(\frac{z}{2}\right)^{-p} \quad \text { for } p \geq 1 .
$$

The property which will interest us here is that $J_{p}(z)$ and $Y_{p}(z)$ form a basis of solutions of the Bessel equation:

$$
\mathcal{D}_{p}^{2} u+u=0, \quad \text { where } \quad \mathcal{D}_{p}^{2} \equiv \frac{1}{z} \frac{d}{d z}\left(z \frac{d}{d z}\right)-\frac{p^{2}}{z^{2}} .
$$

We shall exploit this to introduce two families of functions that will be useful in the next section. First, note that after the shift of index $l \rightarrow l+p$ (for convenience)

$$
J_{p}(z)=\sum_{l=-\infty}^{+\infty} J_{p, p+l}\left(\frac{z}{2}\right)^{p+l}=\sum_{l=-\infty}^{+\infty} \hat{\jmath}_{p, l}\left(\frac{z}{2}\right),
$$

where we have defined

$$
\hat{\jmath}_{p, l}(z)=J_{p, p+l} z^{p+l} .
$$

Substituting $u=J_{p}$ into (3.9), we get, since

$$
\begin{gathered}
\mathcal{D}_{p}^{2} z^{q}=\left(q^{2}-p^{2}\right) z^{q-2} \\
\sum_{l=-\infty}^{+\infty} J_{p, p+l} \frac{(p+l)^{2}-p^{2}}{4}\left(\frac{z}{2}\right)^{p+l-2}+\sum_{l=-\infty}^{+\infty} J_{p, p+l}\left(\frac{z}{2}\right)^{p+l}=0
\end{gathered}
$$

that is,

$$
\left.\sum_{l=-\infty}^{+\infty}\left[J_{p, p+l}\left((p+l)^{2}-p^{2}\right)\right)+4 J_{p, p+l-2}\right]\left(\frac{z}{2}\right)^{p+l-2}=0
$$

which leads to the recurrence equation

$$
\left.J_{p, p+l}\left((p+l)^{2}-p^{2}\right)\right)+4 J_{p, p+l-2}=0,
$$


which is classically used for constructing $J_{p}$ by obtaining (3.5) but can also be rewritten as (multiply (3.14) by $(z / 2)^{p+l-2}$ and use formula (3.12) and the definition (3.11) of $\hat{\jmath}_{p, l}$ )

$$
\mathcal{D}_{p}^{2} \hat{\jmath}_{p, l}+4 \hat{\jmath}_{p, l-2}=0 \quad \forall p, l .
$$

Note that, because of (3.5),

$$
\hat{\jmath}_{p, l} \equiv 0 \quad \text { for negative } l \text { and odd } l .
$$

In particular, for each $p$, the first (with respect to the index $l$ ) nonzero $\hat{\jmath}_{p, l}$ is

$$
\hat{\jmath}_{p, 0}(z)=J_{p, p} z^{p}, \quad \text { and } \quad \mathcal{D}_{p}^{2} \hat{\jmath}_{p, 0}=0 .
$$

In the same way, we can write

$$
Y_{p}(z)=\sum_{l=-\infty}^{+\infty}\left(Y_{p,-p+l}+\frac{2}{\pi} J_{p,-p+l} \log \frac{z}{2}\right)\left(\frac{z}{2}\right)^{-p+l}=\sum_{l=-\infty}^{+\infty} \hat{y}_{p, l}\left(\frac{z}{2}\right),
$$

having defined

$$
\hat{y}_{p, l}(z)=\left(Y_{p,-p+l}+\frac{2}{\pi} J_{p,-p+l} \log z\right) z^{-p+l} .
$$

Then we can use the identity

$$
\mathcal{D}_{p}^{2} z^{q} \log z=z^{q-2}\left(\left(q^{2}-p^{2}\right) \log z+2 q\right)
$$

to substitute $u=Y_{p}$ into (3.9) and obtain

$$
\left\{\begin{aligned}
\frac{1}{4} \sum_{l=-\infty}^{+\infty}\{( & \left.(l-p)^{2}-p^{2}\right) Y_{p,-p+l} \\
+ & {\left.\left[\left((l-p)^{2}-p^{2}\right) \log \frac{z}{2}+2(l-p)\right] \frac{2}{\pi} J_{p,-p+l}\right\}\left(\frac{z}{2}\right)^{-p+l-2} } \\
+ & \sum_{l=-\infty}^{+\infty}\left[Y_{p,-p+l-2}+\frac{2}{\pi} J_{p,-p+l-2} \log \frac{z}{2}\right]\left(\frac{z}{2}\right)^{-p+l-2}=0,
\end{aligned}\right.
$$

which yields, for each $l$,

$$
\left\{\begin{aligned}
&\left((l-p)^{2}-p^{2}\right) Y_{p,-p+l}+\left[\left((l-p)^{2}-p^{2}\right) \log \frac{z}{2}+2(l-p)\right] \frac{2}{\pi} J_{p,-p+l} \\
&+4\left[Y_{p,-p+l-2}+\frac{2}{\pi} J_{p,-p+l-2} \log \frac{z}{2}\right]=0,
\end{aligned}\right.
$$

which is usually exploited to write recurrence relations for the $Y_{p, l}$ 's but that we choose here to reinterpret as the following identity (using (3.12), (3.19), and the definition (3.18) of $\left.\hat{y}_{p, l}\right)$ :

$$
\mathcal{D}_{p}^{2} \hat{y}_{p, l}+4 \hat{y}_{p, l-2}=0 \quad \forall p, l .
$$


Here again, thanks to (3.5) and (3.6),

$$
\hat{y}_{p, l} \equiv 0 \quad \text { for negative } l \text { and odd } l .
$$

In particular, for each $p$, the first (with respect to the index $l$ ) nonzero $\hat{y}_{p, l}$ is

$$
\hat{y}_{p, 0}(z)=\left[Y_{p,-p}+\frac{2}{\pi} J_{p,-p} \log z\right] z^{-p}, \quad \text { and } \quad \mathcal{D}_{p}^{2} \hat{y}_{p, 0}=0 .
$$

3.2.2. Modal expansions of far-fields. To shorten our presentation, let us give some definitions:

- We denote by $H_{0, l o c}^{1}\left(\Omega_{H}\right)$ the space of " $H_{l o c}^{1}\left(\Omega_{H}\right)$ functions except at the origin": ${ }^{2}$

$$
H_{0, l o c}^{1}\left(\Omega_{H}\right)=\left\{u \in \mathcal{D}^{\prime}\left(\Omega_{H}\right) / \forall \varphi \in C_{00}^{\infty}\left(\Omega_{H}\right), \quad \varphi u \in H^{1}\left(\Omega_{H}\right)\right\}
$$

with $C_{00}^{\infty}\left(\Omega_{H}\right)=\left\{\varphi \in C^{\infty}\left(\overline{\Omega_{H}}\right) / \exists r_{1}>r_{0}>0\right.$ such that $r_{0} \geqslant|\mathbf{x}|$ or $\left.|\mathbf{x}| \geqslant r_{1} \Rightarrow \varphi(\mathbf{x})=0\right\}$.

- We will say that $u \in H_{0, l o c}^{1}\left(\Omega_{H}\right)$, such that $\varphi \Delta u \in L^{2}\left(\Omega_{H}\right)$ for all $\varphi \in$ $C_{00}^{\infty}\left(\Omega_{H}\right)$, satisfies the Neumann boundary condition except at the origin:

$$
\frac{\partial u}{\partial n}=0 \text { on } \partial \Omega_{H} \backslash\{\mathbf{0}\}
$$

if and only if

$$
\forall v \in C_{00}^{\infty}\left(\Omega_{H}\right), \quad \int_{\Omega_{H}}(\nabla u(\mathbf{x}) \cdot \nabla v(\mathbf{x})+\Delta u(\mathbf{x}) v(\mathbf{x})) d \mathbf{x}=0 .
$$

Note that as soon as $u \in H_{l o c}^{1}\left(\Omega_{H}\right)$ and $\Delta u \in L_{l o c}^{2}\left(\Omega_{H}\right)$, the above definition corresponds to the classical variational definition of the Neumann condition (by density of $C_{00}^{\infty}\left(\Omega_{H}\right)$ in $H_{l o c}^{1}\left(\Omega_{H}\right)$ ).

- By definition, a far-field is a function $u \in H_{0, l o c}^{1}\left(\Omega_{H}\right)$ that satisfies the homogeneous Helmholtz equation

$$
\Delta u+\omega^{2} u=0 \text { in } \Omega_{H}
$$

and satisfies the Neumann boundary condition on $\partial \Omega_{H} \backslash\{\boldsymbol{0}\}$ in the sense of the above definition (3.25).

REMARK 3.1. The reader will observe that if a given far-field is outgoing at infinity, then it is identically zero as soon as we assume that it belongs to $H_{l o c}^{1}\left(\Omega_{H}\right)$ : this is a consequence of the standard uniqueness theorem for the Helmholtz equation. In other words, nontrivial outgoing far-fields will necessarily be singular at the origin (with a non- $H_{l o c}^{1}$ singularity). The functions $u_{i}^{k}$ of the expansion (2.6) will be of that type.

Next, we introduce the polar coordinates $(r, \theta)$ so that $x=-r \sin \theta, y=r \cos \theta$, and we will be concerned with a space of $H_{0, l o c}^{1}$ functions that satisfy the homogeneous Helmoltz equation and the Neumann condition only in a neighborhood of the origin. More precisely, we introduce

\footnotetext{
${ }^{2}$ The topology on this space is the one generated by the seminorms $\|\varphi u\|_{H^{1}}$ with $\varphi \in C_{00}^{\infty}$.
} 


$$
\left\{\begin{array}{c}
\Omega_{H}^{R}=\left\{\mathbf{x} \in \Omega_{H} \text { and } 0<|\mathbf{x}|<R\right\} \quad(\Longleftrightarrow 0<r<R, 0<\theta<\pi), \\
\mathcal{V}\left(\Omega_{H}^{R}\right)=\left\{u \in H_{0, l o c}^{1}\left(\Omega_{H}\right) / \Delta u+\omega^{2} u=0 \text { in } \Omega_{H}^{R}\right. \\
\text { and } \left.\frac{\partial u}{\partial \theta}(r, \theta=0, \pi)=0,0<r<R\right\} .
\end{array}\right.
$$

Note that $\mathcal{V}\left(\Omega_{H}^{R}\right)$ contains all the far-fields in the sense of the above definition but also the limit field $u_{0}^{0}$ (cf. (3.1)). The method of separation of variables in $(r, \theta)$ naturally introduces the functions $(\cos p \theta, p \in \mathbb{N})$, namely the eigenfunctions of the operator $-d^{2} / d \theta^{2}$ in the interval ] $0, \pi$ [ with Neumann conditions at $\theta=0$ or $\pi$. More precisely, any $u \in \mathcal{V}\left(\Omega_{H}^{R}\right)$ admits the following expansion (where the convergence holds, for instance in $\left.H_{0, l o c}^{1}\left(\Omega_{H}^{R}\right)\right)$ :

$$
u(r, \theta)=\sum_{p=0}^{+\infty}\left(\mathcal{L}_{p}^{0}(u) Y_{p}(\omega r) \cos p \theta+\mathcal{L}_{p}^{1}(u) J_{p}(\omega r) \cos p \theta\right)
$$

where the complex coefficients $\mathcal{L}_{p}^{0}(u)$ and $\mathcal{L}_{p}^{1}(u)$ define linear forms in $\mathcal{V}\left(\Omega_{H}^{R}\right)$ that can be also defined as (setting $\delta_{0}=1$ and $\delta_{p}=2$ for $p>0$ )

$$
\left\{\begin{aligned}
\mathcal{L}_{p}^{0}(u) & =\lim _{r \rightarrow 0} \frac{\delta_{p}}{\pi Y_{p}(\omega r)} \int_{0}^{\pi}[u(r, \theta) \cos p \theta] d \theta \\
\mathcal{L}_{p}^{1}(u) & =\lim _{r \rightarrow 0} \frac{\delta_{p}}{\pi J_{p}(\omega r)} \int_{0}^{\pi}\left[u(r, \theta)-\mathcal{L}_{p}^{0}(u) Y_{p}(\omega r) \cos p \theta\right] \cos p \theta d \theta
\end{aligned}\right.
$$

According to (3.8), the representation formula (3.27) provides a natural decomposition $u=u^{r e g}+u^{\text {sing }}$ as the sum of the regular part $u^{r e g}$ and singular part $u^{\text {sing }}$ of the field $u$, namely

$$
\left\{\begin{array}{l}
u^{r e g}(r, \theta)=\sum_{p=0}^{+\infty} \mathcal{L}_{p}^{1}(u) J_{p}(\omega r) \cos p \theta \\
u^{\operatorname{sing}}(r, \theta)=\sum_{p=0}^{+\infty} \mathcal{L}_{p}^{0}(u) Y_{p}(\omega r) \cos p \theta
\end{array}\right.
$$

REMARK 3.2. One can show [12] that the coefficients $\mathcal{L}_{p}^{1}(u)$ can be written as a linear combination of the $y$ derivatives (the $r$ derivatives for $\theta=0$ ) of $u^{\text {reg }}$ at the point $\mathbf{0}$. For example, one has

$$
\mathcal{L}_{0}^{1}(u)=u^{r e g}(\mathbf{0}), \quad \mathcal{L}_{1}^{1}(u)=\frac{2}{\omega} \frac{\partial u^{r e g}}{\partial y}(\mathbf{0}), \quad \mathcal{L}_{2}^{1}(u)=2 u^{r e g}(\mathbf{0})+\frac{4}{\omega^{2}} \frac{\partial^{2} u^{r e g}}{\partial y^{2}}(\mathbf{0}) .
$$

The degree of singularity of $u$ can be seen from the coefficients $\mathcal{L}_{p}^{0}(u)$.

Definition 3.1. A function $u \in \mathcal{V}\left(\Omega_{H}^{R}\right)$ is singular at order $k \geq 0$ if and only if

$$
\mathcal{L}_{p}^{0}(u)=0 \quad \text { for } p>k .
$$

Remark 3.3. Due to (3.8), we have 


$$
\left\{\begin{aligned}
\mathcal{L}_{p}^{0}(u)=0 \quad \text { for } p>k & \Longleftrightarrow u-\sum_{p=0}^{k} \mathcal{L}_{p}^{0}(u) Y_{p}(\omega r) \cos p \theta \in H_{l o c}^{1}\left(\Omega_{H}\right), \\
& \Longleftrightarrow u(r, \theta)=O\left(r^{-k}\right) \text { if } k>0, \quad=O(\log r) \text { if } k=0
\end{aligned}\right.
$$

REMARK 3.4. If we require the far-field to be outgoing at infinity, it is entirely determined in $\Omega_{H}$ by the knowledge of its singular part $u^{\text {sing }}$ (see, for instance, Lemma 4.2). In particular, the set of outgoing far-fields which are singular of order $k$ at the origin is a vector space of dimension $k+1$ : such a far-field is determined by the knowledge of the $k+1$ complex numbers $\mathcal{L}_{p}^{0}(u)$ for $0 \leq p \leq k$.

3.2.3. Solutions of embedded Laplace equations in $\widehat{\Omega}_{N}$. In this paragraph, we are interested in describing the structure of solutions of embedded Laplace equations in $\widehat{\Omega}_{N}$, i.e., a family of functions $H_{l o c}^{1}\left(\widehat{\Omega}_{N}\right)$ which are inductively related by the equations (with $\mathcal{U}_{-2}=0$ and $\mathcal{U}_{-1}=0$ ):

$$
\Delta \mathcal{U}_{i}=-4 \mathcal{U}_{i-2} \text { in } \widehat{\Omega}_{N} \quad \text { and } \quad \frac{\partial \mathcal{U}_{i}}{\partial n}=0 \text { on } \partial \widehat{\Omega}_{N} .
$$

More precisely, we will give two different modal expansions of the functions $\mathcal{U}_{i}$ in the following two subdomains of $\widehat{\Omega}_{N}$ (see Figure 4.2):

$$
\left\{\begin{array}{l}
\widehat{\Omega}_{N}^{H}=\left\{(X, Y) \in \widehat{\Omega}_{N} / X<0 \text { and } X^{2}+Y^{2}>1\right\} \\
\widehat{\Omega}_{N}^{S}=\left\{(X, Y) \in \widehat{\Omega}_{N} / X>0\right\}
\end{array}\right.
$$

We will use Cartesian coordinates $(X, Y)$ in $\widehat{\Omega}_{N}^{S}$ and polar coordinates $((\rho, \theta), \theta \in$ $[0, \pi])$ in $\widehat{\Omega}_{N}^{H}$ :

$$
X=-\rho \sin \theta \quad \text { and } \quad Y=\rho \cos \theta
$$

Modal expansions in the domain $\widehat{\Omega}_{N}^{H}$. We again use separation of variables in polar coordinates $(\rho, \theta)$. A function $\mathcal{U} \in H_{\text {loc }}^{1}\left(\widehat{\Omega}_{N}^{H}\right)$ can be decomposed in the form

$$
\mathcal{U}(\rho, \theta)=\sum_{p=0}^{+\infty}(\mathcal{U})^{p}(\rho) \cos p \theta
$$

where $(\mathcal{U})^{p}(\rho)=\frac{\delta_{p}}{\pi} \int_{0}^{\pi} \mathcal{U}(\rho, \theta) \cos (p \theta) d \theta$ (with $\delta_{0}=1$ and $\delta_{p}=2$ for $p>0$ ).

Our objective is now to describe more precisely the form of the functions $(\mathcal{U})^{p}(\rho)$ for $\mathcal{U}=\mathcal{U}_{i}$. For $i=0,1, \mathcal{U}_{i}$ is harmonic so that

$$
\forall p \in \mathbb{N}, \quad \mathcal{D}_{p}^{2}\left[\left(\mathcal{U}_{i}\right)^{p}\right]=0 .
$$

According to (3.16) and (3.22), the kernel of the differential operator $\mathcal{D}_{p}^{2}$ is generated by $\hat{\jmath}_{p, 0}(\rho)$ and $\left.\hat{y}_{p, 0}(\rho)\right)$. Thus if we introduce the particular harmonic functions

$$
\left\{\begin{array}{l}
\jmath_{p, 0}(\rho, \theta)=\hat{\jmath}_{p, 0}(\rho) \cos p \theta=J_{p, p} \rho^{p} \cos p \theta \\
y_{p, 0}(\rho, \theta)=\hat{y}_{p, 0}(\rho) \cos p \theta=\left(Y_{p,-p}+\frac{2}{\pi} J_{p,-p} \log \rho\right) \rho^{-p} \cos p \theta
\end{array}\right.
$$


for $i=0,1$, there exist two sequences $\alpha_{i}=\left(\alpha_{i, p}\right)_{p \in \mathbb{N}}$ and $\beta_{i}=\left(\beta_{i, p}\right)_{p \in \mathbb{N}}$ such that

$$
\mathcal{U}_{i}=\sum_{p=0}^{+\infty}\left(\alpha_{i, p} J_{p, 0}+\beta_{i, p} y_{p, 0}\right)
$$

Next, we construct, for each $i$, two particular sequences $\left(\jmath_{p, i}, y_{p, i}, p \in \mathbb{N}\right)$ that are identically 0 for odd $i$ and satisfy embedded equations

$$
\left\{\begin{array}{l}
\Delta j_{p, i}=-4 \jmath_{p, i-2} \text { in } \widehat{\Omega}_{N}^{H} \quad \text { and } \frac{\partial \jmath_{p, i}}{\partial n}=0 \text { on } \partial \widehat{\Omega}_{N}^{H} \cap \partial \widehat{\Omega}_{N} \\
\Delta y_{p, i}=-4 y_{p, i-2} \text { in } \widehat{\Omega}_{N}^{H} \quad \text { and } \frac{\partial y_{p, i}}{\partial n}=0 \text { on } \partial \widehat{\Omega}_{N}^{H} \cap \partial \widehat{\Omega}_{N} .
\end{array}\right.
$$

These functions are constructed from the functions $\left(\hat{\jmath}_{p, i}, \hat{y}_{p, i}\right)$ introduced in section 3.2.1:

$$
\left\{\begin{array}{l}
\jmath_{p, i}(\rho, \theta)=\hat{\jmath}_{p, i}(\rho) \cos p \theta=J_{p, p+i} \rho^{p+i} \cos p \theta, \\
y_{p, i}(\rho, \theta)=\hat{y}_{p, i}(\rho) \cos p \theta=\left(Y_{p,-p+i}+\frac{2}{\pi} J_{p,-p+i} \log \rho\right) \rho^{-p+i} \cos p \theta .
\end{array}\right.
$$

The reader will remark the following:

- The formulas (3.40) define $\jmath_{p, i}$ and $y_{p, i}$ for any $i$ with $\jmath_{p, i}=y_{p, i} \equiv 0$ if $i<0$.

- The log term appears in the definition of $y_{p, i}$ only for $i \geq 2 p\left(J_{p,-p+i}=0\right.$ for $i<2 p$; see $(3.6))$.

- Except for $i=p=0$, the functions $\jmath_{p, i}$ are increasing at infinity while the functions $y_{p, i}$ grow only for $i>p$. In this case, $\jmath_{p, i}$ grows strictly more rapidly than $y_{p, i}$ (except for $p=0$ ).

- Using in particular (3.5) and (3.6), it is possible to show that the functions

$$
\left\{\left(J_{p, i}, y_{p, i}\right) / p \in \mathbb{N}, i \in 2 \mathbb{N}\right\}
$$

are linearly independent.

REMARK 3.5. The introduction of $\jmath_{p, i}$ and $y_{p, i}$ for odd $i$ may appear as a strange choice since they are identically 0 . This is only for convenience and will permit us to write our forthcoming calculations in a more compact form.

Then we are able to prove, by induction on $i$, the following fundamental lemma.

LEMMA 3.2. Let $\left\{\left(\mathcal{U}_{i}\right) / i \in \mathbb{N}\right\}$ be a family of functions in $H_{\text {loc }}^{1}\left(\widehat{\Omega}_{N}\right)$ satisfying (3.33); then there exist two sequences $\left\{\left(\alpha_{p, i}, \beta_{p, i}\right) /(p, i) \in \mathbb{N}^{2}\right\}$ of complex numbers such that

$$
\mathcal{U}_{i}=\sum_{p=0}^{+\infty} \sum_{l=0}^{i}\left(\alpha_{i-l, p} J_{p, l}+\beta_{i-l, p} y_{p, l}\right) \quad \text { in } H_{l o c}^{1}\left(\widehat{\Omega}_{N}^{H}\right)
$$

Proof. First, note that the result is true for $i=0$ and 1 with the sequences $\left(\alpha_{p, 0}, \beta_{p, 0}\right)$ and $\left(\alpha_{p, 1}, \beta_{p, 1}\right)$ introduced previously (see (3.38) and the fact that $\jmath_{p, 1}=$ $\left.y_{p, 1}=0\right)$. By induction, let us admit that the sequences $\left(\alpha_{p, l}, \beta_{p, l}\right)$ have been constructed up to $l=i-1$. Let us introduce (remember that $\jmath_{p, 1}=y_{p, 1}=0$ )

$$
\mathcal{U}_{i}^{*}=\sum_{p=0}^{+\infty} \sum_{l=1}^{i}\left(\alpha_{i-l, p} \jmath_{p, l}+\beta_{i-l, p} y_{p, l}\right) \equiv \sum_{p=0}^{+\infty} \sum_{l=2}^{i}\left(\alpha_{i-l, p} \jmath_{p, l}+\beta_{i-l, p} y_{p, l}\right) .
$$


We have (successively, we use (3.39) and apply the change of index $l \rightarrow l-2$ )

$$
\left\{\begin{aligned}
\Delta \mathcal{U}_{i}^{*} & =\sum_{p=0}^{+\infty} \sum_{l=2}^{i}\left(\alpha_{i-l, p} \Delta \jmath_{p, l}+\beta_{i-l, p} \Delta y_{p, l}\right) \\
& =-4 \sum_{p=0}^{+\infty} \sum_{l=2}^{i}\left(\alpha_{i-l, p} J_{p, l-2}+\beta_{i-l, p} y_{p, l-2}\right) \\
& =-4 \sum_{p=0}^{+\infty} \sum_{l=0}^{i-2}\left(\alpha_{i-2-l, p} J_{p, l}+\beta_{i-2-l, p} y_{p, l}\right)=-4 \mathcal{U}_{i-2}
\end{aligned}\right.
$$

The function $\mathcal{U}_{i}-\mathcal{U}_{i}^{*}$ being harmonic in $\widehat{\Omega}_{N}^{H}$, we know that there exist two sequences of complex numbers, which we choose to denote, respectively, by $\left(\alpha_{i, p}, p \in \mathbb{N}\right)$ and $\left(\beta_{i, p}, p \in \mathbb{N}\right)$, such that

$$
\mathcal{U}_{i}-\mathcal{U}_{i}^{*}=\sum_{p=0}^{+\infty}\left(\alpha_{i, p} \jmath_{p, 0}+\beta_{i, p} y_{p, 0}\right)
$$

and the proof is completed.

REMARK 3.6. Since the proof is done by induction, it is clear that the result of Lemma 3.2 is also valid for finite families $\left\{\mathcal{U}_{i}, i \leqslant N\right\}$, satisfying (3.33) for $i \leq N$, in which case (3.41) holds for $i \leq N$. This remark justifies recurrence proofs that will be done later.

The previous lemma suggests that we introduce the (increasing with $i$ ) subspaces of $H_{l o c}^{1}\left(\widehat{\Omega}_{H}^{N}\right)$ :

$$
\mathcal{V}_{i}\left(\widehat{\Omega}_{N}^{H}\right)=\operatorname{Span}\left\{\left(g_{p, l}, y_{p, l}\right), p \in \mathbb{N}, l \leq i\right\}
$$

(more precisely, the closure in $H_{l o c}^{1}\left(\widehat{\Omega}_{N}^{H}\right)$ of the space generated by the $j_{p}^{l}$ and $y_{p}^{l}$ for $l \leqslant i)$ and

$$
\mathcal{V}\left(\widehat{\Omega}_{N}^{H}\right)=\bigcup_{i=0}^{+\infty} \mathcal{V}_{i}\left(\widehat{\Omega}_{N}^{H}\right)
$$

On $\mathcal{V}\left(\widehat{\Omega}_{N}^{H}\right)$, we can define linear forms $\mathcal{U} \in \mathcal{V}\left(\widehat{\Omega}_{N}^{H}\right) \mapsto \ell_{p}^{0}(\mathcal{U}) \in \mathbb{C}$ and $\mathcal{U} \in \mathcal{V}\left(\widehat{\Omega}_{N}^{H}\right) \mapsto$ $\ell_{p}^{1}(\mathcal{U}) \in \mathbb{C}$ as the coefficients of $\mathcal{U}$ associated with the functions $\left(\jmath_{p, 0}, y_{p, 0}\right)$. More precisely, $\ell_{p}^{0}(\mathcal{U})$ and $\ell_{p}^{1}(\mathcal{U})$ are characterized by

$\forall \mathcal{U} \in \mathcal{V}\left(\widehat{\Omega}_{N}^{H}\right), \quad \mathcal{U}-\sum_{p=0}^{+\infty}\left(\ell_{p}^{0}(\mathcal{U}) \jmath_{p, 0}+\ell_{p}^{1}(\mathcal{U}) y_{p, 0}\right)=\sum_{l \geq 1, \text { finite }} \sum_{p=0}^{+\infty}\left(A_{p, l} \jmath_{p, l}+B_{p, l} y_{p, l}\right)$.

Looking at the expansion $(3.41)$ one sees that $\ell_{p}^{0}\left(\mathcal{U}_{i}\right)=\alpha_{i, p}$ and $\ell_{p}^{1}\left(\mathcal{U}_{i}\right)=\beta_{i, p}$. Thus, the information provided by Lemma 3.2 can be reinterpreted in a form that we state as a corollary.

Corollary 3.3. Let $\left(\mathcal{U}_{i}\right)$ be a family of functions in $H_{\text {loc }}^{1}\left(\widehat{\Omega}_{N}\right)$ satisfying (3.33); then

- the function $\mathcal{U}_{i}$ belongs to $\mathcal{V}_{i}\left(\widehat{\Omega}_{N}^{H}\right)$; 
- it can be expanded as

$$
\mathcal{U}_{i}=\sum_{p=0}^{+\infty} \sum_{l=0}^{i}\left(\ell_{p}^{0}\left(\mathcal{U}_{i-l}\right) \jmath_{p, l}+\ell_{p}^{1}\left(\mathcal{U}_{i-l}\right) y_{p, l}\right) .
$$

REMARK 3.7. Once again, the previous corollary is valid, modulo obvious modifications, for finite families $\mathcal{U}_{i}$ (see also Remark 3.6). Note that (3.48) means that once the functions $\mathcal{U}_{l}$ are given for $l<i$, the coefficients $\left(\ell_{p}^{0}\left(\mathcal{U}_{i}\right), \ell_{p}^{1}\left(\mathcal{U}_{i}\right)\right)$ are the only degrees of freedom for $\mathcal{U}_{i}$, as it was clear in the proof of Lemma 3.2.

Modal expansions in the domain $\widehat{\Omega}_{N}^{S}$. We use the separation of variables in $(X, Y)$. Let us introduce the functions

$$
\psi_{p}(Y)=\cos (p \pi(Y+1 / 2)), \quad p \in \mathbb{N},
$$

namely the eigenfunctions of the operator $-d^{2} / d Y^{2}$ in the interval $]-1 / 2,+1 / 2[$ with Neumann conditions at $Y= \pm 1 / 2$. Any function $\mathcal{U} \in H_{\text {loc }}^{1}\left(\widehat{\Omega}_{N}^{S}\right)$ can be decomposed in the form

$$
\mathcal{U}(X, Y)=\sum_{p=0}^{+\infty}[\mathcal{U}]_{p}(X) \psi_{p}(Y)
$$

This is in particular the case for each function $\mathcal{U}_{i}$. For $i=0$ and $1, \mathcal{U}_{i}$ is harmonic and therefore

$$
\left[\mathcal{U}_{i}\right]_{p}^{\prime \prime}-p^{2} \pi^{2}\left[\mathcal{U}_{i}\right]_{p}=0
$$

Hence, there exist two sequences $\gamma_{p, i}$ and $\delta_{p, i}$ such that

$$
\mathcal{U}_{i}=\sum_{p=0}^{+\infty}\left[\gamma_{p, i} c_{p, 0}(X, Y)+\delta_{p, i} d_{p, 0}(X, Y)\right]
$$

where $c_{p, 0}$ and $d_{p, 0}$ are given by

$$
\begin{cases}c_{p, 0}(X, Y)=e^{+p \pi X} \psi_{p}(Y) & \text { for } p>0, \quad c_{0,0}(X, Y)=2 \mathbf{i} X \\ d_{p, 0}(X, Y)=e^{-p \pi X} \psi_{p}(Y) & \text { for } p \in \mathbb{N}\end{cases}
$$

where, for the definition of $c_{0,0}$, the factor $2 \mathbf{i}$ has been introduced for convenience. Now we introduce two families of functions $c_{p, i}$ and $d_{p, i}$ for $p \in \mathbb{N}$ and $i \in \mathbb{N}^{*}$, which are identically 0 for $i$ odd and satisfy by construction

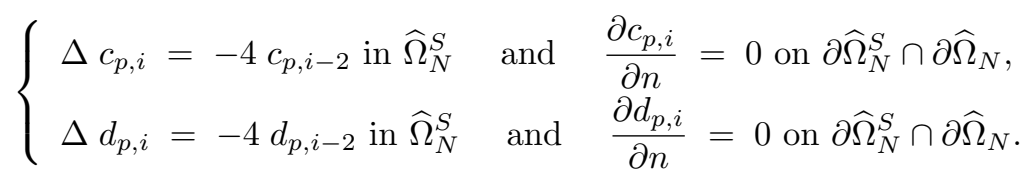

These functions are given by

$$
\left\{\begin{array}{l}
c_{p, i}(X, Y)=\widehat{c}_{p, i}(X) e^{+p \pi X} \psi_{p}(Y), \\
d_{p, i}(X, Y)=\widehat{d}_{p, i}(X) e^{-p \pi X} \psi_{p}(Y),
\end{array}\right.
$$


where the functions $\widehat{c}_{p, i}$ and $\widehat{d}_{p, i}$ are uniquely defined by

$$
\begin{cases}\widehat{c}_{p, i}^{\prime \prime}+2 \pi p \widehat{c}_{p, i}^{\prime}=-4 \widehat{c}_{p, i-2}\left(\equiv(2 \mathbf{i})^{2} \widehat{c}_{p, i-2}\right), & \widehat{c}_{p, i}(0)=\widehat{c}_{p, i}^{\prime}(0)=0 \\ \widehat{d}_{p, i}^{\prime \prime}-2 \pi p \widehat{d}_{p, i}^{\prime}=-4 \widehat{d}_{p, i-2}\left(\equiv(2 \mathbf{i})^{2} \widehat{d}_{p, i-2}\right), & \widehat{d}_{p, i}(0)=\widehat{d}_{p, i}^{\prime}(0)=0\end{cases}
$$

Starting (see (3.53)) from $\widehat{c}_{0,0}(X)=2 \mathbf{i} X, \widehat{c}_{p, 0}(X)=1$ for $p \geq 1$ and $\widehat{d}_{p, 0}(X)=1$ for all $p$. By induction on $i$, one easily sees that

$$
\forall i \geq 0, \quad\left(\widehat{c}_{0, i}, \widehat{d}_{0, i}\right) \in \mathbb{P}^{i+1} \times \mathbb{P}^{i}, \quad \text { and } \quad\left(\widehat{c}_{p, i}, \widehat{d}_{p, i}\right) \in \mathbb{P}^{i} \times \mathbb{P}^{i} \quad \forall p \in \mathbb{N}^{*},
$$

where $\mathbb{P}^{i}$ is the space of polynomials of degree less than or equal to $i$. In what follows, we shall not need the explicit expression of $\widehat{c}_{p, i}$ and $\widehat{d}_{p, i}$ except for $p=0$ for which one easily shows that

$$
\widehat{c}_{0,2 l}(X)=\frac{(2 \mathbf{i} X)^{2 l+1}}{(2 l+1) !}, \quad \widehat{d}_{0,2 l}(X)=\frac{(2 \mathbf{i} X)^{2 l}}{(2 l) !} .
$$

We then state the following lemma, whose proof is identical to the one of Lemma 3.2.

LEMma 3.4. Let $\left(\mathcal{U}_{i}\right)$ be a family of functions in $H_{\text {loc }}^{1}\left(\widehat{\Omega}_{N}\right)$ satisfying (3.33); then there exist two sequences $\left(\gamma_{p, i}, \delta_{p, i}\right)$ of complex numbers such that

$$
\mathcal{U}_{i}=\sum_{p=0}^{+\infty} \sum_{l=0}^{i}\left[\gamma_{p, i-l} c_{p, l}+\delta_{p, i-l} d_{p, l}\right] \quad \text { in } H_{l o c}^{1}\left(\widehat{\Omega}_{N}^{S}\right)
$$

As in the previous paragraph, we introduce ${ }^{3}$

$$
\mathcal{V}_{i}\left(\widehat{\Omega}_{N}^{S}\right)=\operatorname{Span}\left\{\left(c_{p, l} ; d_{p, l}\right), p \in \mathbb{N}, l \leqslant i\right\}, \quad \mathcal{V}\left(\widehat{\Omega}_{N}^{S}\right)=\bigcup_{i=0}^{+\infty} \mathcal{V}_{i}\left(\widehat{\Omega}_{N}^{S}\right)
$$

and linear forms $\mathcal{U} \in \mathcal{V}\left(\widehat{\Omega}_{N}^{S}\right) \mapsto L_{p}^{0}(\mathcal{U}) \in \mathbb{C}$ and $\mathcal{U} \in \mathcal{V}\left(\widehat{\Omega}_{N}^{S}\right) \mapsto L_{p}^{1}(\mathcal{U}) \in \mathbb{C}$ such that

$\forall \mathcal{U} \in \mathcal{V}\left(\widehat{\Omega}_{N}^{s}\right), \quad \mathcal{U}-\sum_{p=0}^{+\infty}\left(L_{p}^{0}(\mathcal{U}) c_{p, 0}+L_{p}^{1}(\mathcal{U}) d_{p, 0}\right)=\sum_{l \geq 1, \text { finite }} \sum_{p=0}^{+\infty}\left(C_{p, l} c_{p, l}+D_{p, l} d_{p, l}\right)$

We can then rewrite Lemma 3.4 as follows.

Corollary 3.5. Let $\left(\mathcal{U}_{i}\right)$ be a family of functions in $H_{\text {loc }}^{1}\left(\widehat{\Omega}_{N}\right)$ satisfying (3.33); then

- the function $\mathcal{U}_{i}$ belongs to $\mathcal{V}_{i}\left(\widehat{\Omega}_{N}^{S}\right)$;

- it can be expanded as

$$
\mathcal{U}_{i}=\sum_{p=0}^{+\infty} \sum_{l=0}^{i}\left(L_{p}^{0}\left(\mathcal{U}_{i-l}\right) c_{p, l}+L_{p}^{1}\left(\mathcal{U}_{i-l}\right) d_{p, l}\right)
$$

Remark 3.8. Here again, Lemma 3.4 and Corollary 3.5 are valid for finite families $\mathcal{U}_{i}$ (in the sense explained in Remark 3.6).

\footnotetext{
${ }^{3}$ More precisely, the closure in $H_{l o c}^{1}\left(\widehat{\Omega}_{N}^{S}\right)$ of the space generated by the $c_{p}^{l}$ and $d_{p}^{l}$ for $l \leqslant i$.
} 
3.3. Presentation of the coupled problems defining $u_{i}^{k}, U_{i}^{k}, \mathcal{U}_{i}^{k}$ for $i>0$. For indices $(i, k) \notin \mathbf{J}$ (see (2.4) and Figure 2.1), we adopt the following convention:

$$
u_{i}^{k} \equiv 0, \quad \mathcal{U}_{i}^{k} \equiv 0, \quad U_{i}^{k} \equiv 0 \quad \forall(i, k) \notin \mathbf{J} .
$$

The problem to be solved is the following:

Find the family of functions $u_{i}^{k} \in H_{0, l o c}^{1}\left(\Omega_{H}\right), \mathcal{U}_{i}^{k} \in H_{l o c}^{1}\left(\widehat{\Omega}_{N}\right)$, and $U_{i}^{k} \in H_{l o c}^{1}\left(\mathbb{R}^{+}\right)$ with $(i, k) \in \mathbf{J} \backslash\{(0,0)\}$ such that the following hold:

- For each $(i, k)$, the function $u_{i}^{k}$ belongs to $\mathcal{V}\left(\Omega_{H}^{R}\right)$ and is a singular outgoing solution of the Helmholtz equation in $\Omega_{H}$ satisfying

$$
\left\{\begin{array}{l}
\Delta u_{i}^{k}+\omega^{2} u_{i}^{k}=0 \text { in } \Omega_{H} \quad \text { and } \quad \frac{\partial u_{i}^{k}}{\partial n}=0 \text { on } \partial \Omega_{H} \backslash\{\mathbf{0}\} \\
\mathcal{L}_{p}^{0}\left(u_{i}^{k}\right)=0 \quad \forall p>i-k .
\end{array}\right.
$$

- The functions $\mathcal{U}_{i}^{k}$ belong to $\mathcal{V}\left(\widehat{\Omega}_{N}^{H}\right) \cap \mathcal{V}\left(\widehat{\Omega}_{N}^{S}\right)$ and are, for each $k$, solutions of nonhomogeneous embedded Laplace equations

$$
\left\{\begin{array}{l}
\Delta \mathcal{U}_{i}^{k}=-4 \mathcal{U}_{i-2}^{k} \text { in } \widehat{\Omega}_{N} \quad \text { and } \quad \frac{\partial \mathcal{U}_{i}^{k}}{\partial n}=0 \text { on } \partial \widehat{\Omega}_{N}, \\
\ell_{p}^{0}\left(\mathcal{U}_{i}^{k}\right)=0 \quad \forall p>i-k \\
L_{p}^{0}\left(\mathcal{U}_{i}^{k}\right)=0 \quad \forall p>0 .
\end{array}\right.
$$

- For each $(i, k)$, the function $U_{i}^{k}$ is an outgoing solution of the 1D Helmholtz equation in $\mathbb{R}^{+}$, namely

$$
U_{i}^{k}(x)=U_{i}^{k}(0) \exp \mathbf{i} \omega x .
$$

- The behaviors of $u_{i}^{k}$ at the origin and of $\mathcal{U}_{i}^{k}$ at infinity in $\widehat{\Omega}_{N}^{H}$ are linked by the conditions

$$
\begin{cases}\mathcal{L}_{p}^{0}\left(u_{i}^{k}\right)=\ell_{p}^{1}\left(\mathcal{U}_{i-p}^{k}\right) & \text { for } 0 \leqslant p \leqslant i-k, \\ \ell_{p}^{0}\left(\mathcal{U}_{i}^{k}\right)=\mathcal{L}_{p}^{1}\left(u_{i-p}^{k}\right)+\frac{2}{\pi} \mathcal{L}_{p}^{0}\left(u_{i-p}^{k-1}\right) & \text { for } 0 \leqslant p \leqslant i-k .\end{cases}
$$

- The behavior at infinity of $\mathcal{U}_{i}^{k}$ in $\widehat{\Omega}_{N}^{S}$ is linked to the behavior at the origin of $U_{i}^{k}$ and $U_{i-1}^{k}$, namely

$$
L_{0}^{0}\left(\mathcal{U}_{i}^{k}\right)=U_{i-1}^{k}(0) \quad \text { and } \quad L_{0}^{1}\left(\mathcal{U}_{i}^{k}\right)=U_{i}^{k}(0) .
$$

The reader can refer to (3.26) (3.45), (3.46), and (3.60) for the definition of the spaces $\mathcal{V}\left(\Omega_{H}^{R}\right), \mathcal{V}\left(\widehat{\Omega}_{N}^{H}\right), \mathcal{V}\left(\widehat{\Omega}_{N}^{S}\right)$. In these spaces we can define the linear forms $\mathcal{L}_{p}^{0}, \mathcal{L}_{p}^{1}, \ell_{p}^{0}, \ell_{p}^{1}$, $L_{p}^{0}$, and $L_{p}^{1}$, which give the spatial asymptotic expansions of $u_{i}^{k}$ and $\mathcal{U}_{i}^{k}$ via (3.29), (3.48), and (3.62).

Remark 3.9. Conditions (3.64(b)), (3.65(b)), and (3.65(c)) and (3.68) give (nonoptimal; see Remark 4.3) information on the far-field singularity and on the 
near-field growing in the half-space and in the slot. The conditions (3.64(b)) could be replaced by the following: $u_{i}^{k}$ is singular at order $i-k$ (see Definition 3.1). The conditions $(3.65(\mathrm{~b}))$ are equivalent to $\mathcal{U}_{i}^{k}(\rho, \theta)=O\left(\rho^{i-k} \log \rho\right)$ when $\rho \rightarrow+\infty$. Indeed, the modal expansion (3.48) (with $\mathcal{U}_{i-l}^{k} \equiv 0$ for $l>i-k$ ) leads to

$$
\mathcal{U}_{i}^{k}=\sum_{p=0}^{+\infty} \sum_{l=0}^{i-k}\left(\ell_{p}^{0}\left(\mathcal{U}_{i-l}^{k}\right) \jmath_{p, l}\right)+\sum_{p=0}^{+\infty} \sum_{l=0}^{i-k}\left(\ell_{p}^{1}\left(\mathcal{U}_{i-l}^{k}\right) y_{p, l}\right) .
$$

Due to $(3.65(\mathrm{~b}))$, one has $\ell_{p}^{0}\left(\mathcal{U}_{i-l}^{k}\right)=0$ for $p>i-l-k$ :

$$
\mathcal{U}_{i}^{k}=\sum_{l=0}^{i-k} \sum_{p=0}^{i-k-l}\left(\ell_{p}^{0}\left(\mathcal{U}_{i-l}^{k}\right) \jmath_{p, l}\right)+\sum_{p=0}^{+\infty} \sum_{l=0}^{i-k}\left(\ell_{p}^{1}\left(\mathcal{U}_{i-l}^{k}\right) y_{p, l}\right) .
$$

As $p+l$ is smaller than $i-k$ in the first sum, this yields when $\rho \rightarrow+\infty$ to

$$
\mathcal{U}_{i}^{k}=O\left(\rho^{i-k}\right)+O\left(\rho^{i-k} \log \rho\right) \quad\left(j_{p, l}=O\left(\rho^{p+l}\right) \text { and } y_{p, l}=O\left(\rho^{-p+l} \log \rho\right)\right) .
$$

One can also easily see that $(3.65(\mathrm{c}))$ and (3.68) lead to $\mathcal{U}_{i}^{k}=O\left(X^{i-k}\right)$ when $X \rightarrow$ $+\infty$. We say that $\mathcal{U}_{i}^{k}$ is growing at infinity at order $i-k$.

3.4. Derivation of the coupled problems. In this section, we are going to derive (3.64), (3.65), (3.66), (3.67), and (3.68).

Derivation of $(3.64(\mathrm{a})),(3.65(\mathrm{a}))$, and (3.66). The calculations are straightforward and the details left to the reader.

- (3.64(a)) follows immediately from substituting (2.6) into the Helmholtz equation (and Neumann condition) written in $\Omega_{H}(\varepsilon)$. The fact that $u_{i}$ is outgoing follows in the same way.

- (3.65(a)) is obtained by substituting the ansatz (2.12) into the Helmholtz equation (and Neumann condition) written in $\Omega_{N}(\varepsilon)$.

- Finally, substituting (2.8) into the Helmholtz equation (and Neumann condition) written in $\Omega_{S}(\varepsilon)$, we get

$$
\left\{\begin{array}{l}
-\frac{\omega^{2}}{4} \frac{\partial^{2} U_{i}^{k}}{\partial Y^{2}}=\frac{\partial^{2} U_{i-2}^{k}}{\partial x^{2}}+\omega^{2} U_{i-2}^{k} \quad \text { in } \widehat{\Omega}_{S}, \\
\frac{\partial U_{i}^{k}}{\partial Y}\left(x,-\frac{1}{2}\right)=\frac{\partial U_{i}^{k}}{\partial Y}\left(x, \frac{1}{2}\right)=0, \quad x>0 .
\end{array}\right.
$$

Setting $\widetilde{U}_{i}^{k}(x)=\int_{-1 / 2}^{1 / 2} U_{i}^{k}(x, Y) d Y$, after integration of (3.72) over $Y$, we easily get

$$
\frac{\partial^{2} \widetilde{U}_{i}^{k}}{\partial x^{2}}+\omega^{2} \widetilde{U}_{i}^{k}=0, \quad x>0,
$$

which leads, using the outgoing condition in the slot (we omit the details), to

$$
\widetilde{U}_{i}^{k}(x)=\widetilde{U}_{i}^{k}(0) \exp \mathbf{i} \omega x
$$

To conclude, it suffices to show that, for any $i \geq k, U_{i}^{k}$ is constant in $Y$ so that $U_{i}^{k}=\widetilde{U}_{i}^{k}$. This is immediate for $i=k, k+1\left(\partial^{2} U_{i}^{k} / \partial Y^{2}\right.$ and $\partial U_{i}^{k} / \partial Y$ vanish for $Y=-1 / 2$ and $1 / 2$ ) and obtained by induction for $i \geq 2$. 
Matching between far-field and near-field: Derivation of (3.64(b)), (3.65(b)), and (3.67). Here we choose to derive the matching condition using the identification of formal series. However, the alternative Van Dyke matching principle (see [5]), modified adequately to take into account the presence of the logarithmic terms, leads to the same result.

We consider the overlapping zone in $\Omega_{\varepsilon}$ for near- and far-fields:

$$
\Omega_{H}(\varepsilon) \cap \Omega_{N}(\varepsilon)=\left\{(x, y) \in \Omega_{\varepsilon} / x<0, \quad \eta_{H}^{-}(\varepsilon) \leqslant r \leqslant \eta_{H}^{+}(\varepsilon)\right\} .
$$

In this zone, we express $u^{\varepsilon}$ via our two ansatzes (2.6) and (2.12), written in polar coordinates:

$\sum_{i=0}^{+\infty} \sum_{k=0}^{i}\left(\frac{\omega \varepsilon}{2}\right)^{i} \log ^{k}\left(\frac{\omega \varepsilon}{2}\right) u_{i}^{k}(r, \theta)=u^{\varepsilon}(r, \theta)=\sum_{i=0}^{+\infty} \sum_{k=0}^{i}\left(\frac{\omega \varepsilon}{2}\right)^{i} \log ^{k}\left(\frac{\omega \varepsilon}{2}\right) \mathcal{U}_{i}^{k}\left(\frac{r}{\varepsilon}, \theta\right)$.

In the rest of this section, we shall write infinite sums over $i \in]-\infty ;+\infty[, k \in$ ]$-\infty ;+\infty[, p \in[0 ;+\infty[$, and $l \in]-\infty ;+\infty[$. This has the advantage of simplifying the manipulations of infinite sums and is completely justified, as the reader can easily check, thanks to our convention (3.63). First, thanks to (3.64(a)), we can expand spatially the functions $u_{i}^{k}$ via (3.27). Then, denoting by $L$ the left-hand side of (3.74),

$$
L=\sum_{i, k, p}\left(\frac{\omega \varepsilon}{2}\right)^{i} \log ^{k}\left(\frac{\omega \varepsilon}{2}\right)\left(\mathcal{L}_{p}^{0}\left(u_{i}^{k}\right) Y_{p}(\omega r)+\mathcal{L}_{p}^{1}\left(u_{i}^{k}\right) J_{p}(\omega r)\right) \cos p \theta
$$

For the right-hand side $R$ of (3.74), we expand the functions $\mathcal{U}_{i}^{k}$ using (3.47) and Corollary 3.3 , which can be applied to each family $i \rightarrow \mathcal{U}_{i}^{k}$ thanks to (3.65(a)). We get

$$
R=\sum_{i, k, p, l}\left(\frac{\omega \varepsilon}{2}\right)^{i} \log ^{k}\left(\frac{\omega \varepsilon}{2}\right)\left(\ell_{p}^{0}\left(\mathcal{U}_{i-l}^{k}\right) \jmath_{p, l}\left(\frac{r}{\varepsilon}, \theta\right)+\ell_{p}^{1}\left(\mathcal{U}_{i-l}^{k}\right) y_{p, l}\left(\frac{r}{\varepsilon}, \theta\right)\right)
$$

Using the definitions of the functions $\jmath_{p, l}$ and $y_{p, l}$ (see (3.40), we get

$$
\left\{\begin{aligned}
R & =\sum_{i, k, p, l}\left(\frac{\omega \varepsilon}{2}\right)^{i} \log ^{k}\left(\frac{\omega \varepsilon}{2}\right) \ell_{p}^{0}\left(\mathcal{U}_{i-l}^{k}\right) J_{p, p+l}\left(\frac{r}{\varepsilon}\right)^{p+l} \cos p \theta \\
& +\sum_{i, k, p, l}\left(\frac{\omega \varepsilon}{2}\right)^{i} \log ^{k}\left(\frac{\omega \varepsilon}{2}\right) \ell_{p}^{1}\left(\mathcal{U}_{i-l}^{k}\right)\left(Y_{p,-p+l}+\frac{2}{\pi} \log \frac{r}{\varepsilon} J_{p,-p+l}\right)\left(\frac{r}{\varepsilon}\right)^{-p+l} \cos p \theta \\
& =\sum_{i, k, p, l}\left(\frac{\omega \varepsilon}{2}\right)^{i-l-p} \log ^{k}\left(\frac{\omega \varepsilon}{2}\right) \ell_{p}^{0}\left(\mathcal{U}_{i-l}^{k}\right) J_{p, p+l}\left(\frac{\omega r}{2}\right)^{p+l} \cos p \theta \\
& +\sum_{i, k, p, l}\left(\frac{\omega \varepsilon}{2}\right)^{i-l+p} \log ^{k}\left(\frac{\omega \varepsilon}{2}\right) \ell_{p}^{1}\left(\mathcal{U}_{i-l}^{k}\right)\left(Y_{p,-p+l}+\frac{2}{\pi} \log \frac{\omega r}{2} J_{p,-p+l}\right)\left(\frac{\omega r}{2}\right)^{-p+l} \cos p \theta \\
& -\sum_{i, k, p, l}\left(\frac{\omega \varepsilon}{2}\right)^{i-l+p} \log ^{k+1}\left(\frac{\omega \varepsilon}{2}\right) \ell_{p}^{1}\left(\mathcal{U}_{i-l}^{k}\right) \frac{2}{\pi} J_{p,-p+l}\left(\frac{\omega r}{2}\right)^{-p+l} \cos p \theta,
\end{aligned}\right.
$$

where we have used $\log (r / \varepsilon)=\log (\omega r / 2)-\log (\omega \varepsilon / 2)$. Applying, for the summation over $i$, the change of index $i \rightarrow i-l-p$ in the first sum and $i \rightarrow i-l+p$ in the last 
two, we obtain

$$
\left\{\begin{aligned}
R & =\sum_{i, k, p, l}\left(\frac{\omega \varepsilon}{2}\right)^{i} \log ^{k}\left(\frac{\omega \varepsilon}{2}\right) \ell_{p}^{0}\left(\mathcal{U}_{i+p}^{k}\right) J_{p, p+l}\left(\frac{\omega r}{2}\right)^{p+l} \cos p \theta \\
& +\sum_{i, k, p, l}\left(\frac{\omega \varepsilon}{2}\right)^{i} \log ^{k}\left(\frac{\omega \varepsilon}{2}\right) \ell_{p}^{1}\left(\mathcal{U}_{i-p}^{k}\right)\left(Y_{p,-p+l}+\frac{2}{\pi} \log \frac{\omega r}{2} J_{p,-p+l}\right)\left(\frac{\omega r}{2}\right)^{-p+l} \cos p \theta \\
& -\sum_{i, k, p, l}\left(\frac{\omega \varepsilon}{2}\right)^{i} \log ^{k}\left(\frac{\omega \varepsilon}{2}\right) \ell_{p}^{1}\left(\mathcal{U}_{i-p}^{k-1}\right) \frac{2}{\pi} J_{p,-p+l}\left(\frac{\omega r}{2}\right)^{-p+l} \cos p \theta
\end{aligned}\right.
$$

We recognize in the sums over $l$ the expansions (see $(3.4)$ ) of $J_{p}(\omega r)$ and $Y_{p}(\omega r)$. Thus

$$
\begin{aligned}
R & =\sum_{i, k, p}\left(\frac{\omega \varepsilon}{2}\right)^{i} \log ^{k}\left(\frac{\omega \varepsilon}{2}\right) \\
& \times\left[\left(\ell_{p}^{0}\left(\mathcal{U}_{i+p}^{k}\right)-\frac{2}{\pi} \ell_{p}^{1}\left(\mathcal{U}_{i-p}^{k-1}\right)\right) J_{p}(\omega r)+\ell_{p}^{1}\left(\mathcal{U}_{i-p}^{k}\right) Y_{p}(\omega r)\right] \cos p \theta
\end{aligned}
$$

Identifying the terms of the two series $L$ and $R$ leads to

$$
\mathcal{L}_{p}^{0}\left(u_{i}^{k}\right)=\ell_{p}^{1}\left(\mathcal{U}_{i-p}^{k}\right) \quad \text { and } \quad \mathcal{L}_{p}^{1}\left(u_{i}^{k}\right)=\ell_{p}^{0}\left(\mathcal{U}_{i+p}^{k}\right)-\frac{2}{\pi} \ell_{p}^{1}\left(\mathcal{U}_{i-p}^{k-1}\right)
$$

or equivalently to the coupling conditions

$$
\mathcal{L}_{p}^{0}\left(u_{i}^{k}\right)=\ell_{p}^{1}\left(\mathcal{U}_{i-p}^{k}\right) \quad \text { and } \quad \ell_{p}^{0}\left(\mathcal{U}_{i}^{k}\right)=\mathcal{L}_{p}^{1}\left(u_{i-p}^{k}\right)+\frac{2}{\pi} \mathcal{L}_{p}^{0}\left(u_{i-p}^{k-1}\right) \quad \forall i, k, p .
$$

The matching conditions (3.67) are nothing but (3.78) specified to $0 \leq l \leq i-k$.

The conditions $(3.64(\mathrm{~b}))\left(u_{i}^{k}\right.$ is singular at order $\left.i-k\right)$ are obtained by remarking that, for $p>i-k$,

$$
\mathcal{L}_{p}^{0}\left(u_{i}^{k}\right)=\ell_{p}^{1}\left(\mathcal{U}_{i-p}^{k}\right)=0
$$

since $i-p<k$ means that $(i-p, k)$ does not belong to $\mathbf{J}$ and thus $\mathcal{U}_{i-p}^{k}=0$.

The conditions $(3.65(\mathrm{~b}))$ also follow since

- if $p>i-k+1,(i-p, k)$ and $(i-p, k-1)$ do not belong to $J$ so that

$$
u_{i-p}^{k}=u_{i-p}^{k-1} \equiv 0 \Longrightarrow \ell_{p}^{0}\left(\mathcal{U}_{i}^{k}\right)=0(\operatorname{see}(3.78))
$$

- if $p=i-k+1,(i-p, k)$ does not belong to $J$ so that

$$
u_{i-p}^{k} \equiv 0 \Longrightarrow \ell_{p}^{0}\left(\mathcal{U}_{i}^{k}\right)=\frac{2}{\pi} \mathcal{L}_{i-k+1}^{0}\left(u_{k-1}^{k-1}\right)=\frac{2}{\pi} \ell_{i-k+1}^{1}\left(\mathcal{U}_{k-1-(i-k+1)}^{k-1}\right)(\operatorname{see}(3.78)) .
$$

As $(i, k) \in \mathbf{J}, i-k+1$ is strictly positive. Hence, $(k-1-(i-k+1), k-1)$ is not in $\mathbf{J}$ :

$$
\mathcal{U}_{k-1-(i-k+1)}^{k-1} \equiv 0 \quad \Longrightarrow \quad \ell_{p}^{0}\left(\mathcal{U}_{i}^{k}\right)=0
$$

Matching between near-field and slot-field: Derivation of (3.65(c)) and (3.68). We consider the overlapping zone in $\Omega_{\varepsilon}$ for near- and slot-fields:

$$
\Omega_{N}(\varepsilon) \cap \Omega_{S}(\varepsilon)=\left\{(x, y) \in \Omega_{\varepsilon} / x>0, \quad \eta_{S}^{-}(\varepsilon) \leqslant x \leqslant \eta_{S}^{+}(\varepsilon)\right\} .
$$


In this zone, we express $u^{\varepsilon}$ via our two ansatzes (2.8) and (2.12), written in Cartesian coordinates (using again the convention (3.63)):

$\sum_{i, k}\left(\frac{\omega \varepsilon}{2}\right)^{i} \log ^{k}\left(\frac{\omega \varepsilon}{2}\right) \mathcal{U}_{i}^{k}\left(\frac{x}{\varepsilon}, \frac{y}{\varepsilon}\right)=u^{\varepsilon}(x, y)=\sum_{i, k}\left(\frac{\omega \varepsilon}{2}\right)^{i} \log ^{k}\left(\frac{\omega \varepsilon}{2}\right) U_{i}^{k}(x)+O\left(\varepsilon^{\infty}\right)$.

Denoting by $R$ the right-hand side of (3.81), we have (3.82), using (3.66) and replacing exp $i \omega x$ by its series expansion (the reason for writing the second line below will appear later),

$$
\left\{\begin{aligned}
R & =\sum_{i, k, l}\left(\frac{\omega \varepsilon}{2}\right)^{i} \log ^{k}\left(\frac{\omega \varepsilon}{2}\right) U_{i}^{k}(0) \frac{(i \omega x)^{l}}{l !}+O\left(\varepsilon^{\infty}\right) \\
& =\sum_{i, k, l}\left(\frac{\omega \varepsilon}{2}\right)^{i} \log ^{k}\left(\frac{\omega \varepsilon}{2}\right) U_{i}^{k}(0)\left[\frac{(i \omega x)^{2 l+1}}{(2 l+1) !}+\frac{(i \omega x)^{2 l}}{2 l !}\right]+O\left(\varepsilon^{\infty}\right) .
\end{aligned}\right.
$$

Next, we expand the left-hand side $L$ of (3.74) using (3.62) of Corollary 3.5, which can be applied to each family $i \rightarrow \mathcal{U}_{i}^{k}$ thanks to $(3.65(\mathrm{a}))$. We get

$$
L=\sum_{i, k, p, l}\left(\frac{\omega \varepsilon}{2}\right)^{i} \log ^{k}\left(\frac{\omega \varepsilon}{2}\right)\left[L_{p}^{0}\left(\mathcal{U}_{i-l}^{k}\right) c_{p, l}\left(\frac{x}{\varepsilon}, \frac{y}{\varepsilon}\right)+L_{p}^{1}\left(\mathcal{U}_{i-l}^{k}\right) d_{p, l}\left(\frac{x}{\varepsilon}, \frac{y}{\varepsilon}\right)\right]
$$

Noticing that for $p>0$ the quantities $d_{p, l}\left(\frac{x}{\varepsilon}, \frac{y}{\varepsilon}\right)$ are $O\left(\varepsilon^{\infty}\right)$ terms in $\Omega_{N}(\varepsilon) \cap \Omega_{S}(\varepsilon)$, this can be rewritten as

$$
\left\{\begin{aligned}
L & =\sum_{i, k, l}\left(\frac{\omega \varepsilon}{2}\right)^{i} \log ^{k}\left(\frac{\omega \varepsilon}{2}\right)\left[L_{0}^{0}\left(\mathcal{U}_{i-l}^{k}\right) \widehat{c}_{0, l}\left(\frac{x}{\varepsilon}\right)+L_{0}^{1}\left(\mathcal{U}_{i-l}^{k}\right) \widehat{d}_{0, l}\left(\frac{x}{\varepsilon}\right)\right] \\
& +\sum_{p \geq 1} \sum_{i, k, l}\left(\frac{\omega \varepsilon}{2}\right)^{i} \log ^{k}\left(\frac{\omega \varepsilon}{2}\right) L_{p}^{0}\left(\mathcal{U}_{i-l}^{k}\right) \widehat{c}_{p, l}\left(\frac{x}{\varepsilon}\right) \psi_{p}\left(\frac{y}{\varepsilon}\right) e^{p \pi x / \varepsilon}+O\left(\varepsilon^{\infty}\right)
\end{aligned}\right.
$$

where we have isolated in the expansion the term which is a function of $x$ only. Using the expression of $\widehat{c}_{0, l}$ and $\widehat{d}_{0, l}$, we thus have

$$
\left\{\begin{aligned}
L & =\sum_{i, k, l}\left(\frac{\omega \varepsilon}{2}\right)^{i} \log ^{k}\left(\frac{\omega \varepsilon}{2}\right)\left[L_{0}^{0}\left(\mathcal{U}_{i-2 l}^{k}\right) \frac{(2 \mathbf{i} x / \varepsilon)^{2 l+1}}{(2 l+1) !}+L_{0}^{1}\left(\mathcal{U}_{i-2 l}^{k}\right) \frac{(2 \mathbf{i} x / \varepsilon)^{2 l}}{2 l !}\right] \\
& +\sum_{p \geq 1} \sum_{i, k, l}\left(\frac{\omega \varepsilon}{2}\right)^{i} \log ^{k}\left(\frac{\omega \varepsilon}{2}\right) L_{p}^{0}\left(\mathcal{U}_{i-l}^{k}\right) \widehat{c}_{p, l}\left(\frac{x}{\varepsilon}\right) \psi_{p}\left(\frac{y}{\varepsilon}\right) e^{p \pi x / \varepsilon}+O\left(\varepsilon^{\infty}\right) \\
& =\sum_{i, k, l}\left(\frac{\omega \varepsilon}{2}\right)^{i-2 l-1} \log ^{k}\left(\frac{\omega \varepsilon}{2}\right) L_{0}^{0}\left(\mathcal{U}_{i-2 l}^{k}\right) \frac{(\mathbf{i} \omega x)^{2 l+1}}{(2 l+1) !} \\
& +\sum_{i, k, l}\left(\frac{\omega \varepsilon}{2}\right)^{i-2 l} \log ^{k}\left(\frac{\omega \varepsilon}{2}\right) L_{0}^{1}\left(\mathcal{U}_{i-2 l}^{k}\right) \frac{(\mathbf{i} \omega x)^{2 l}}{2 l !} \\
& +\sum_{p \geq 1} \sum_{i, k, l}\left(\frac{\omega \varepsilon}{2}\right)^{i} \log ^{k}\left(\frac{\omega \varepsilon}{2}\right) L_{p}^{0}\left(\mathcal{U}_{i-l}^{k}\right) \widehat{c}_{p, l}\left(\frac{x}{\varepsilon}\right) \psi_{p}\left(\frac{y}{\varepsilon}\right) e^{p \pi x / \varepsilon}+O\left(\varepsilon^{\infty}\right)
\end{aligned}\right.
$$


or equivalently, after change of index in the sum over $i$,

$$
\left\{\begin{aligned}
L & =\sum_{i, k, l}\left(\frac{\omega \varepsilon}{2}\right)^{i} \log ^{k}\left(\frac{\omega \varepsilon}{2}\right)\left[L_{0}^{0}\left(\mathcal{U}_{i+1}^{k}\right) \frac{(\mathbf{i} \omega x)^{2 l+1}}{(2 l+1) !}+L_{0}^{1}\left(\mathcal{U}_{i}^{k}\right) \frac{(\mathbf{i} \omega x)^{2 l}}{2 l !}\right] \\
& +\sum_{p \geq 1} \sum_{i, k, l}\left(\frac{\omega \varepsilon}{2}\right)^{i} \log ^{k}\left(\frac{\omega \varepsilon}{2}\right) L_{p}^{0}\left(\mathcal{U}_{i-l}^{k}\right) \widehat{c}_{p, l}\left(\frac{x}{\varepsilon}\right) \psi_{p}\left(\frac{y}{\varepsilon}\right) e^{p \pi x / \varepsilon}+O\left(\varepsilon^{\infty}\right)
\end{aligned}\right.
$$

The identification of (3.82) and (3.86) leads to

$$
\left\{\begin{array}{l}
\forall i, k, \quad L_{0}^{0}\left(\mathcal{U}_{i+1}^{k}\right)=L_{0}^{1}\left(\mathcal{U}_{i}^{k}\right)=U_{i}^{k}(0), \\
\forall i, k \quad \forall p \geq 1, \quad L_{p}^{0}\left(\mathcal{U}_{i}^{k}\right)=0
\end{array}\right.
$$

This is nothing but (3.65(c)) and (3.68).

4. Existence and uniqueness result. The object of this paragraph is to prove the following result.

THEOREM 4.1. There exists a unique family of functions $u_{i}^{k}, \mathcal{U}_{i}^{k}$, and $U_{i}^{k}$ for $(i, k) \in \mathbf{J} \backslash(0,0)$ satisfying (3.64), (3.65), (3.66), (3.67), and (3.68). Moreover, $u_{i}^{i}=0$ for all $i \geq 1$.

Preliminary step. The first step is to eliminate the $U_{i}^{k}$ 's using (3.68). One sees that if $\left(\left(u_{i}^{k}, \mathcal{U}_{i}^{k}, U_{i}^{k}\right),(i, k) \in \mathbf{J}\right)$ is solution of ((3.64), (3.65), (3.66), (3.67), (3.68)), then $\left(\left(u_{i}^{k}, \mathcal{U}_{i}^{k}\right),(i, k) \in \mathbf{J}\right)$ is solution of the following problem:

Find $u_{i}^{k} \in H_{0, l o c}^{1}\left(\Omega_{H}\right)$ outgoing and $\mathcal{U}_{i}^{k} \in H_{l o c}^{1}\left(\widehat{\Omega}_{N}\right)$ such that

$$
\begin{aligned}
& \left\{\begin{array}{l}
\Delta u_{i}^{k}+\omega^{2} u_{i}^{k}=0 \quad \text { in } \Omega_{H} \quad \text { and } \quad \frac{\partial u_{i}^{k}}{\partial n}=0 \quad \text { on } \partial \Omega_{H} \backslash\{\mathbf{0}\} \\
\mathcal{L}_{p}^{0}\left(u_{i}^{k}\right)=0 \quad \text { for } p>i-k
\end{array}\right.
\end{aligned}
$$

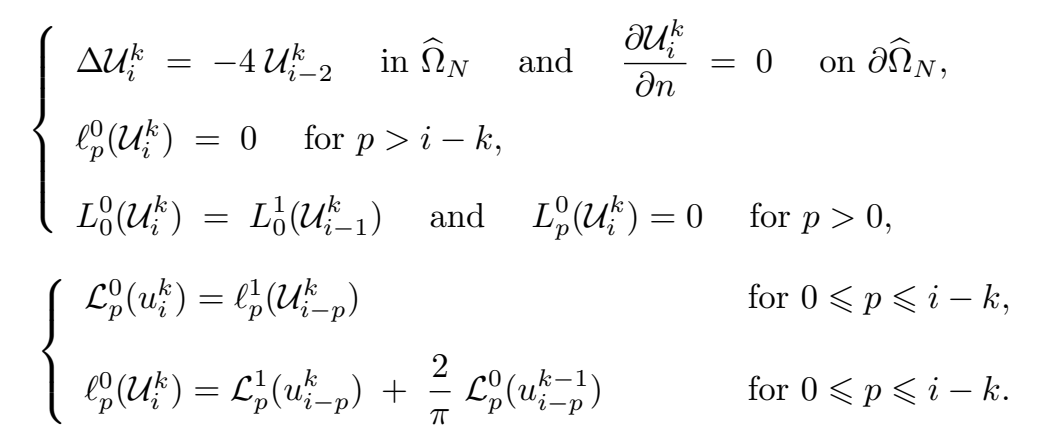

Reciprocally, one checks easily that if $\left(\left(u_{i}^{k}, \mathcal{U}_{i}^{k}\right),(i, k) \in \mathbf{J}\right)$ is solution of the problem ((4.1), (4.2), (4.3)), then, defining

$$
U_{i}^{k}(x)=L_{0}^{1}\left(\mathcal{U}_{i}^{k}\right) \exp \mathbf{i} \omega \mathbf{x},
$$

$\left(\left(u_{i}^{k}, \mathcal{U}_{i}^{k}, U_{i}^{k}\right),(i, k) \in \mathbf{J}\right)$ is solution of $((3.64),(3.65),(3.66),(3.67),(3.68))$.

Uniqueness. By induction on $(i, k)$, it is easy to see that the uniqueness result will be obtained if we are able to prove that the unique solution $(u, \mathcal{U})$ of the following problem: 
Find $u \in H_{0, l o c}^{1}\left(\Omega_{H}\right)$ outgoing and $\mathcal{U} \in H_{l o c}^{1}\left(\widehat{\Omega}_{N}\right)$ such that

$$
\begin{aligned}
& \left\{\begin{array}{l}
\Delta u+\omega^{2} u=0 \text { in } \Omega_{H} \quad \text { and } \quad \frac{\partial u}{\partial n}=0 \text { on } \partial \Omega_{H} \backslash\{\mathbf{0}\} \\
\mathcal{L}_{p}^{0}(u)=0 \text { for } p>0
\end{array}\right. \\
& \left\{\begin{array}{l}
\Delta \mathcal{U}=0 \text { in } \widehat{\Omega}_{N} \text { and } \frac{\partial \mathcal{U}}{\partial n}=0 \text { on } \partial \widehat{\Omega}_{N}, \\
\ell_{p}^{0}(\mathcal{U})=0 \quad \text { for } p>0 \\
L_{p}^{0}(\mathcal{U})=0 \quad \text { for } p \in \mathbb{N}
\end{array}\right. \\
& \left\{\begin{array}{l}
\mathcal{L}_{0}^{0}(u)=\ell_{0}^{1}(\mathcal{U}) \\
\ell_{0}^{0}(\mathcal{U})=\mathcal{L}_{0}^{1}(u)
\end{array}\right.
\end{aligned}
$$

is the trivial solution $u=\mathcal{U} \equiv 0$.

To show that $u=0$, we remark from (4.5) that it suffices to prove that $\mathcal{L}_{0}^{0}(u)=0$. Indeed, this will imply that $u$ is a regular outgoing far-field and is thus identically 0 (see Remark 3.1). From the first equation of (4.7), we see that it suffices to show that $\ell_{0}^{1}(\mathcal{U})=0$.

Since $\mathcal{U}$ is solution of a homogeneous Laplace equation in $\widehat{\Omega}_{N}$ with homogeneous Neumann conditions, we can write its modal expansion in $\widehat{\Omega}_{N}^{H}$, using (4.6), as follows (see (3.48) with $i=0)$ :

$$
\mathcal{U}=\sum_{p=0}^{+\infty}\left(\ell_{p}^{0}(\mathcal{U}) \jmath_{p, 0}+\ell_{p}^{1}(\mathcal{U}) y_{p, 0}\right)=\ell_{0}^{0}(\mathcal{U}) \jmath_{0,0}+\sum_{p=0}^{+\infty} \ell_{p}^{1}(\mathcal{U}) y_{p, 0}
$$

Next, the key remark is that, for $p>0$, both $\jmath_{p, 0}$ and $y_{p, 0}$ have mean value 0 with respect to $\theta$ varying in $] 0, \pi[$. Therefore, for any $\rho>1$ we have

$$
\frac{1}{\pi} \int_{0}^{\pi} \mathcal{U}(\rho, \theta) d \theta=\left(\frac{1}{\pi} \int_{0}^{\pi} \jmath_{0,0}(\rho, \theta) d \theta\right) \ell_{0}^{0}(\mathcal{U})+\left(\frac{1}{\pi} \int_{0}^{\pi} y_{0,0}(\rho, \theta) d \theta\right) \ell_{0}^{1}(\mathcal{U})
$$

that is, using the expression (3.37) of $\jmath_{0,0}$ and $y_{0,0}$

$$
\frac{1}{\pi} \int_{0}^{\pi} \mathcal{U}(\rho, \theta) d \theta=\ell_{0}^{1}(\mathcal{U})\left(Y_{0,0}+\frac{2}{\pi} J_{0,0} \log \rho\right)+\ell_{0}^{0}(\mathcal{U}) J_{0,0} \quad \forall \rho>1 .
$$

By differentiation with respect to $\rho$, we get rid of the term in $\ell_{0}^{0}(\mathcal{U})$ and obtain, since $J_{0,0}=1$,

$$
\int_{0}^{\pi} \frac{\partial \mathcal{U}}{\partial \rho}(\rho, \theta) d \theta=\frac{2}{\rho} \ell_{0}^{1}(\mathcal{U}) \quad \Longrightarrow(\rho \rightarrow 1) \quad \ell_{0}^{1}(\mathcal{U})=\frac{1}{2} \int_{0}^{\pi} \frac{\partial \mathcal{U}}{\partial \rho}(1, \theta) d \theta
$$

Then, to obtain $\ell_{0}^{0}(\mathcal{U})$, we integrate the Laplace equation over the domain (see Figure 4.1)

$$
\widehat{\Omega}_{N}^{I}=\left\{(\rho, \theta) \in \Omega_{N} / 0 \leqslant \rho \leqslant 1 \text { and } 0 \leqslant \theta \leqslant \pi\right\},
$$




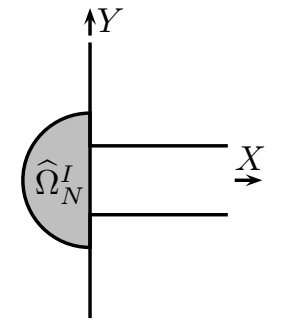

FIG. 4.1. The domain $\widehat{\Omega}_{N}^{I}$.

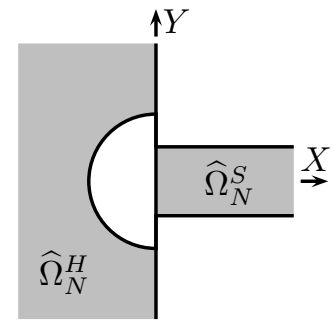

FIG. 4.2. The domain $\widehat{\Omega}_{N}^{H}$ and $\widehat{\Omega}_{N}^{S}$.

which, using the Green-Riemann formula, leads to

$$
\int_{\widehat{\Omega}_{N}^{I}} \Delta \mathcal{U}=\int_{\partial \hat{\Omega}_{N}^{I}} \frac{\partial \mathcal{U}}{\partial n} \Longleftrightarrow \int_{0}^{\pi} \frac{\partial \mathcal{U}}{\partial \rho}(1, \theta) d \theta+\int_{-\frac{1}{2}}^{\frac{1}{2}} \frac{\partial \mathcal{U}}{\partial X}(0, Y) d Y=0 .
$$

Moreover, since $\mathcal{U}$ is harmonic in $\widehat{\Omega}_{N}^{S}$ and satisfies $\frac{\partial \mathcal{U}}{\partial n}=0$ on $\partial \widehat{\Omega}_{N}$, we know (see Lemma 3.4) that the function

$$
X \rightarrow \int_{-\frac{1}{2}}^{\frac{1}{2}} \mathcal{U}(X, Y) d Y
$$

is affine. More precisely,

$$
\int_{-\frac{1}{2}}^{\frac{1}{2}} \mathcal{U}(X, Y) d Y=L_{0}^{1}(\mathcal{U})+2 \mathbf{i} L_{0}^{0}(\mathcal{U}) X=L_{0}^{1}(\mathcal{U}),
$$

where we have used the last equation of (4.6). Therefore, after differentiation in $X$

$$
\int_{-\frac{1}{2}}^{\frac{1}{2}} \frac{\partial \mathcal{U}}{\partial X}(0, Y) d Y=0 \Longrightarrow(\operatorname{using}(4.11) \text { and }(4.13)) \quad \ell_{0}^{1}(\mathcal{U})=0,
$$

which we wanted to show. Once $u=0$, we observe, using the first equation of (4.6), that $\mathcal{U}$ satisfies

$$
\begin{cases}\Delta \mathcal{U}=0 \text { in } \widehat{\Omega}_{N} & \text { and } \quad \frac{\partial \mathcal{U}}{\partial n}=0 \text { on } \partial \widehat{\Omega}_{N}, \\ \ell_{p}^{0}(\mathcal{U})=0 & \text { for } p \in \mathbb{N} \quad \text { and } \quad \ell_{0}^{1}(\mathcal{U})=0 \\ L_{p}^{0}(\mathcal{U})=0 & \text { for } p \in \mathbb{N} .\end{cases}
$$

Therefore, we see that $\mathcal{U}$ admits the following expansions (using (3.48) and (3.62) with $i=0$; see Figure 4.2):

$$
\begin{cases}\mathcal{U}=\sum_{p=1}^{+\infty} \ell_{p}^{1}(\mathcal{U}) y_{p, 0} & \text { in } \widehat{\Omega}_{N}^{H} \\ \mathcal{U}=\sum_{p=0}^{+\infty} L_{p}^{1}(\mathcal{U}) d_{p, 0} & \text { in } \widehat{\Omega}_{N}^{S}\end{cases}
$$


Since the functions $y_{p, 0}$ for $p>0$ (resp., $d_{p, 0}$ for $p>0$ ) decay at infinity in $\widehat{\Omega}_{N}^{H}$ (resp., $\left.\widehat{\Omega}_{N}^{S}\right)$ and since $d_{0,0}=1$, this yields

$$
\lim _{\rho \rightarrow+\infty} \sup _{\theta \in[0, \pi]}|\mathcal{U}(\rho, \theta)|=0 \quad \text { and } \quad \mathcal{U} \text { is bounded in } \widehat{\Omega}_{N}^{S} .
$$

The rigorous proof requires us to pay attention to the infinite series. This is where the $H_{l o c}^{1}$ regularity comes in and permits us to get uniform bounds. We refer the reader to [12] for more details.

It has been shown in [7] (see Lemma A.1) that this implies that $\mathcal{U} \equiv 0$.

Existence. With the Lemmas 4.2 and 4.3, we introduce some "basis functions" (defined as solutions of uncoupled problems) in order to reduce the problem to a finite dimensional one.

Lemma 4.2. For all $p \in \mathbb{N}$, there exists a unique $w_{p} \in H_{0, l o c}^{1}\left(\Omega_{H}\right)$ satisfying

$$
\left\{\begin{array}{l}
\Delta w_{p}+\omega^{2} w_{p}=0 \text { in } \Omega_{H} \quad \text { and } \quad \frac{\partial w_{p}}{\partial n}=0 \text { on } \partial \Omega_{H} \backslash\{\mathbf{0}\} \\
\mathcal{L}_{q}^{0}\left(w_{p}\right)=1 \text { if } p=q \quad \text { and } \quad \mathcal{L}_{q}^{0}\left(w_{p}\right)=0 \text { else. }
\end{array}\right.
$$

Proof. Due to (3.32), we first remark that the second line of (4.18) is equivalent to

$$
w_{p}-Y_{p}(\omega r) \cos (p \theta) \in H_{l o c}^{1}\left(\Omega_{H}\right) .
$$

Then, to prove the existence and uniqueness of $w_{p}$, we introduce the new unknown:

$$
w_{p}^{r e g}(r, \theta)=w_{p}(r, \theta)-\chi(r) Y_{p}(\omega r) \cos p \theta
$$

with $\chi$ a $C^{\infty}$ cut-off function equal to 1 near $r=0$ and equal to 0 for $r \geqslant R$. The function $w_{p}^{r e g}$ satisfies a standard Helmholtz problem with Neumann boundary condition and source term compactly supported in the open $\Omega_{H}$. Classical theorems apply.

REMARK 4.1. In the special case where there is no obstacle in the half-space $(\mathcal{B}=\emptyset)$, the $w_{p}$ are known explicitly:

$$
w_{p}(r, \theta)=\frac{1}{\mathbf{i}} H_{p}^{(1)}(\omega r) \cos p \theta \text { with } H_{p}^{(1)}(z)=J_{p}(z)+\mathbf{i} Y_{p}(z) .
$$

Lemma 4.3. For all $p \in \mathbb{N}$, there exists a unique family of functions $\mathcal{W}_{p, l} \in$ $H_{\text {loc }}^{1}\left(\widehat{\Omega}_{N}\right) \cap \mathcal{V}\left(\widehat{\Omega}_{N}^{H}\right) \cap \mathcal{V}\left(\widehat{\Omega}_{N}^{S}\right)$, with $l \in \mathbb{N}$, satisfying

$\left\{\begin{array}{l}\Delta \mathcal{W}_{p, l}=-4 \mathcal{W}_{p, l-2} \text { in } \widehat{\Omega}_{N} \quad \text { and } \quad \frac{\partial \mathcal{W}_{p, l}}{\partial n}=0 \text { on } \partial \widehat{\Omega}_{N} \quad\left(\mathcal{W}_{p, l} \equiv 0 \text { for } l<0\right) \\ \ell_{q}^{0}\left(\mathcal{W}_{p, l}\right)=1 \text { if } q=p \text { and } l=0 \quad \text { and } \quad \ell_{q}^{0}\left(\mathcal{W}_{p, l}\right)=0 \text { else }, \\ L_{0}^{0}\left(\mathcal{W}_{p, l}\right)=L_{0}^{1}\left(\mathcal{W}_{p, l-1}\right) \quad \text { and } \quad L_{q}^{0}\left(\mathcal{W}_{p, l}\right)=0 \quad \text { for } q>0 .\end{array}\right.$

Moreover, one has for $p$ in $\mathbb{N}$

$$
\ell_{0}^{1}\left(\mathcal{W}_{p, 0}\right)=0
$$


The proof of this lemma is more delicate and is postponed to the appendix.

Remark 4.2. One can easily check that $\mathcal{W}_{0,0} \equiv 1$. Moreover, one can obtain more information about the $\mathcal{W}_{p, 0}$ and $\mathcal{W}_{p, 1}$ for $p \leq 1$ using conformal mapping techniques (see [21]).

We can now proceed to a constructive existence proof (that can also be used as an algorithm for numerical computations). To shorten the presentation, we have chosen to introduce some formalism, taking the risk to add some abstraction. However, we hope this will not hide the idea of the proof which is simple: it consists of looking for each $u_{i}^{k}$ (resp., $\mathcal{U}_{i}^{k}$ ) as a particular (finite) linear combination of the $w_{p}$ 's (resp., $\mathcal{W}_{p, l}$ 's) defined above.

We shall work with sequences with three indices:

$$
\mathbf{a}=\left\{\left(a_{i}^{k}\right)_{p} \in \mathbb{C} /(i, k) \in \mathbf{J} \backslash\{(0,0)\} \text { and } p \in \mathbb{N}\right\}
$$

and we shall denote by $\mathbf{S}$ the subset of such sequences such that

$$
\mathbf{S}=\left\{\mathbf{a}=\left(\left(a_{i}^{k}\right)_{p}\right) /\left(a_{i}^{k}\right)_{p}=0 \text { for } p>i-k\right\} .
$$

To any $\mathbf{a} \in \mathbf{S}$, we shall associate, respectively,

$$
\begin{cases}a^{k}=\left\{\left(a_{i}^{k}\right)_{p} \in \mathbb{C} / p \in \mathbb{N} \text { and } i \geqslant k\right\}, & \text { a "two indices"-sequence, } \\ a_{i}^{k}=\left\{\left(a_{i}^{k}\right)_{p} \in \mathbb{C} / p \in \mathbb{N}\right\}, & \text { a "one index"-sequence. }\end{cases}
$$

Note that, due to (4.24), each sequence $a_{i}^{k}$ can be identified to a vector of $\mathbb{C}^{i-k+1}$.

To a sequence $\mathbf{a} \in \mathbf{S}$, we also associate the family of functions in $H_{l o c}^{1}\left(\Omega_{H}\right)$ defined by

$$
u\left(a_{i}^{k}\right)=\sum_{p=0}^{+\infty}\left(a_{i}^{k}\right)_{p} w_{p}, \quad(i, j) \in \mathbf{J} \backslash\{(0,0)\} \quad \text { (due to (4.24), the sum is finite). }
$$

By definition of the $w_{p}$ 's, each $u\left(a_{i}^{k}\right)$ is an outgoing far-field in the sense of section 3.2 .2 and

$$
\mathcal{L}_{p}^{0}\left(u\left(a_{i}^{k}\right)\right)=\left(a_{i}^{k}\right)_{p} \quad \forall(i, k) \in \mathbf{J} \backslash\{(0,0)\}, \forall p \in \mathbb{N} .
$$

To another sequence $\mathbf{A} \in \mathbf{S}$, we associate the family of functions in $H_{l o c}^{1}\left(\widehat{\Omega}_{N}\right)$ defined by

$\mathcal{U}_{i}\left(A^{k}\right)=\sum_{p=0}^{+\infty} \sum_{l=0}^{i-k}\left(A_{i-l}^{k}\right)_{p} \mathcal{W}_{p, l}, \quad(i, k) \in \mathbf{J} \backslash\{(0,0)\} \quad$ (once again the sum is finite),

where, by convention, we have set (this is needed for defining $\mathcal{U}_{i}\left(A^{k}\right)$ when $i$ and $k$ are zeros)

$$
\left(A_{0}^{0}\right)_{0}=u_{0}^{0}(\mathbf{0}) \quad \text { and } \quad\left(A_{0}^{0}\right)_{p}=0 \quad \forall p>0 .
$$

Note that this convention permits us to extend the validity of the formula (4.28) to $i=k=0$. Indeed, this gives $\mathcal{U}_{0}\left(A^{0}\right)=u_{0}^{0}(\mathbf{0}) \mathcal{W}_{0,0}$ with $\mathcal{W}_{0,0}=1$ (see $(3.2)$ ). 
By definition of the $\mathcal{W}_{p, l}$ 's, each family $i \rightarrow \mathcal{U}_{i}\left(A^{k}\right)$ obeys embedded Laplace equations in $\widehat{\Omega}_{H}^{N}$ with Neumann boundary conditions on $\partial \widehat{\Omega}_{H}^{N}$. Indeed,

$$
\Delta \mathcal{U}_{i}\left(A^{k}\right)=\sum_{p=0}^{+\infty} \sum_{l=0}^{i-k}\left(A_{i-l}^{k}\right)_{p} \Delta \mathcal{W}_{p, l}=-4 \sum_{p=0}^{+\infty} \sum_{l=0}^{i-k}\left(A_{i-l}^{k}\right)_{p} \mathcal{W}_{p, l-2}
$$

Making the change of index $l-2 \rightarrow l$ and exploiting $\mathcal{W}_{p, l}=0$ for $l<0$, we get

$$
\Delta \mathcal{U}_{i}\left(A^{k}\right)=-4 \sum_{p=0}^{+\infty} \sum_{l=0}^{i-k-2}\left(A_{i-2-l}^{k}\right)_{p} \mathcal{W}_{p, l}(\rho, \theta)=-4 \mathcal{U}_{i-2}\left(A^{k}\right)
$$

Moreover, by construction of the $\mathcal{W}_{p, l}$ 's, one has

$$
\ell_{p}^{0}\left(\mathcal{U}_{i}\left(A^{k}\right)\right)=\left(A_{i}^{k}\right)_{p} \quad \forall(i, k) \in \mathbf{J} \backslash\{(0,0)\}, \forall p \in \mathbb{N},
$$

and, by manipulations similar to the ones we applied to obtain (4.31), one shows that

$$
L_{0}^{0}\left(\mathcal{U}_{i}\left(A^{k}\right)\right)=L_{0}^{1}\left(\mathcal{U}_{i-1}\left(A^{k}\right)\right) .
$$

The idea is now to construct adequately the sequences $\mathbf{a}$ and $\mathbf{A}$ in $\mathbf{S}$ so that

$$
u_{i}^{k} \equiv u\left(a_{i}^{k}\right) \quad \text { and } \quad \mathcal{U}_{i}^{k} \equiv \mathcal{U}_{i}\left(A^{k}\right) \quad \forall(i, k) \in \mathbf{J} \backslash\{(0,0)\}
$$

are solutions to (4.1), (4.2), and (4.3).

We first notice that, whatever the choice of $\mathbf{a}$ and $\mathbf{A}$ in $\mathbf{S},(4.1)$ and (4.2) are automatically satisfied. It remains to ensure the coupling condition (4.3), which will define inductively on $(i, k)$ the sequences $a_{i}^{k}$ and $A_{i}^{k}$.

Assume that the sequences $a_{j}^{l}$ and $A_{j}^{l}$ have been determined for $(j, l)<(i, k)$ in such a way that equations (4.3) (after having replaced $(i, k)$ by $(j, l)$ ) are satisfied, for all $(j, l)<(i, k)$, by $u_{j}^{l}=u\left(a_{j}^{l}\right)$ and $\mathcal{U}_{j}^{l}=\mathcal{U}_{j}\left(A^{l}\right)$. We explain below how (4.3) leads to the construction of $a_{i}^{k}$ and $A_{i}^{k}$. In the rest of this paragraph, we set, for simplicity, $u_{i}^{k}=u\left(a_{i}^{k}\right)$ and $\mathcal{U}_{i}^{k}=\mathcal{U}_{i}\left(A^{k}\right)$.

If $i>k$, we begin by determining the $\left(a_{i}^{k}\right)_{p}$ 's and $\left(A_{i}^{k}\right)_{p}$ 's $0<p \leq i-k$. We note that writing (4.3) for $0<p \leq i-k$ gives, taking into account (4.27) and (4.32),

$$
\left(a_{i}^{k}\right)_{p}=\ell_{p}^{1}\left(\mathcal{U}_{i-p}^{k}\right) \quad \text { and } \quad\left(A_{i}^{k}\right)_{p}=\mathcal{L}_{p}^{1}\left(u_{i-p}^{k}\right)+\frac{2}{\pi} \mathcal{L}_{p}^{0}\left(u_{i-p}^{k-1}\right),
$$

which determines the $\left(a_{i}^{k}\right)_{p}$ 's and the $\left(A_{i}^{k}\right)_{p}$ 's from the previous $(j, l)$ 's since $(i-p, k)<$ $(i, k)$.

In any case (i.e., even if $i=k$ ), it remains only to determine $\left(a_{i}^{k}\right)_{0}$ and $\left(A_{i}^{k}\right)_{0}$ in order to satisfy (4.3) for $p=0$. Using again (4.27) and (4.32), the first equation of (4.3) with $p=0$ gives

$$
\left(a_{i}^{k}\right)_{0}=\mathcal{L}_{0}^{0}\left(u_{i}^{k}\right)=\ell_{0}^{1}\left(\mathcal{U}_{i}^{k}\right)=\sum_{p=0}^{+\infty} \sum_{l=0}^{i-k}\left(A_{i-l}^{k}\right)_{p} \ell_{0}^{1}\left(\mathcal{W}_{p, l}\right) .
$$

As, for all $p>0, \ell_{0}^{1}\left(\mathcal{W}_{p, 0}\right)=0$ (see (4.23) in Lemma 4.3), this can be rewritten

$$
\left(a_{i}^{k}\right)_{0}=\sum_{p=0}^{+\infty} \sum_{l=1}^{i-k}\left(A_{i-l}^{k}\right)_{p} \ell_{0}^{1}\left(\mathcal{W}_{p, l}\right) \quad \text { for } 0 \leqslant k \leqslant i-1 \text { and }\left(a_{i}^{i}\right)_{0}=0
$$


which determines the $\left(a_{i}^{k}\right)_{0}$ 's from the previous $(j, l)$ 's since $(i-l, k)<(i, k)$ for $l>0$.

Finally, by (4.32), the second equation of (4.3) gives

$$
\left(A_{i}^{k}\right)_{0}=\sum_{p=0}^{i-k}\left(a_{i}^{k}\right)_{p} \mathcal{L}_{0}^{1}\left(w_{p}\right)+\frac{2}{\pi} \mathcal{L}_{0}^{0}\left(u_{i}^{k-1}\right) .
$$

This concludes the proof of existence and uniqueness of the $u_{i}^{k}$ 's and $\mathcal{U}_{i}^{k}$ 's. Moreover, an immediate consequence of (4.26) and (4.36) is $u_{k}^{k} \equiv 0$ for all $k \neq 0$.

REMARK 4.3. One can prove (see [12]) that, for $i>k$, the functions $u_{i}^{k}$, a priori singular at order $i-k$, are in fact only singular at order $i-k-1$ (this is what we announced in section $2 ;$ see (2.14)). In the same way, one shows with the same type of arguments that the growth at infinity of $\mathcal{U}_{i}^{k}$ in $\widehat{\Omega}_{N}^{H}$ is less than the $O\left(\rho^{i-k} \log \rho\right)$ growth predicted by Remark 3.9. More precisely, one shows that (2.17) holds.

Appendix. Proof of Lemma 4.3. Let us recall first an existence and uniqueness result for a Laplace equation in the domain $\widehat{\Omega}_{N}$ with prescribed behavior at infinity. This result is proven in [7].

Lemma A.1. Let $\mathcal{U}_{H} \in H_{l o c}^{1}\left(\widehat{\Omega}_{N}^{H}\right), \mathcal{U}_{S} \in H_{l o c}^{1}\left(\widehat{\Omega}_{N}^{S}\right)$, and $f \in L_{l o c}^{2}\left(\widehat{\Omega}_{N}\right)$ such that

$$
\left\{\begin{array}{llll}
\Delta \mathcal{U}_{H}=-f & \text { in } \widehat{\Omega}_{N}^{H}, & \frac{\partial \mathcal{U}_{H}}{\partial x}=0 & \text { for } x=0, \\
\Delta \mathcal{U}_{S}=-f & \text { in } \widehat{\Omega}_{N}^{S}, & \frac{\partial \mathcal{U}_{S}}{\partial y}=0 & \text { for } y=0 \text { or } y=1 .
\end{array}\right.
$$

There exists a unique $\mathcal{U} \in H_{\text {loc }}^{1}\left(\widehat{\Omega}_{N}\right)$ such that

$$
\left\{\begin{array}{l}
\Delta \mathcal{U}=-f \quad \text { in } \widehat{\Omega}_{N}, \quad \frac{\partial \mathcal{U}}{\partial n}=0 \quad \text { on } \partial \widehat{\Omega}_{N} \\
\lim _{\rho \rightarrow+\infty} \sup _{\theta \in[0 ; \pi]}\left|\mathcal{U}(\rho, \theta)-\mathcal{U}_{H}(\rho, \theta)\right|=0 \\
\mathcal{U}-\mathcal{U}_{S} \text { is bounded in } \widehat{\Omega}_{N}^{S}
\end{array}\right.
$$

if and only if

$$
\int_{0}^{\pi} \frac{\partial \mathcal{U}_{H}}{\partial \rho}(1, \theta) d \theta+\int_{-\frac{1}{2}}^{\frac{1}{2}} \frac{\partial \mathcal{U}_{S}}{\partial x}(0, Y) d Y+\int_{\widehat{\Omega}_{N}^{I}} f d x=0
$$

where $\widehat{\Omega}_{N}^{H}, \widehat{\Omega}_{N}^{S}$, and $\widehat{\Omega}_{N}^{I}$ are defined in (3.34) and (4.12).

REMARK A.1. The condition (A.3) is analogous to the classical compatibility condition for the nonhomogeneous Neumann problem for $-\Delta$ in bounded domains. The proof of Lemma A.1 consists of reducing (A.2) to a similar problem with homogeneous conditions at infinity. This is done by subtracting from $\mathcal{U}$ an appropriate function which is constructed with the help of $\mathcal{U}_{H}$ and $\mathcal{U}_{S}$ and an adequate truncation process. Then the homogeneous conditions are handled through a variational formulation in weighted Sobolev spaces. Finally, the use of Hardy's inequalities permits us to show that the Lax-Milgram lemma can be applied to the new problem.

For $p \in \mathbb{N}$ given, the functions $\mathcal{W}_{p, l}$ will be defined by induction over $l$, using Lemma A.1.

Construction of $\mathcal{W}_{p, \boldsymbol{l}}$ for $\boldsymbol{l}=\mathbf{0}$. According to Lemma 4.3, we look for $\mathcal{W}_{p, 0}$ solution of

$$
\Delta \mathcal{W}_{p, 0}=0 \text { in } \widehat{\Omega}_{N} \quad \text { and } \quad \frac{\partial \mathcal{W}_{p, 0}}{\partial n}=0 \text { on } \partial \widehat{\Omega}_{N}
$$


and satisfying

$$
\left\{\begin{array}{l}
\ell_{q}^{0}\left(\mathcal{W}_{p, 0}\right)=1 \text { if } q=p \quad \text { and } \quad \ell_{q}^{0}\left(\mathcal{W}_{p, 0}\right)=0 \text { else } \\
L_{q}^{0}\left(\mathcal{W}_{p, 0}\right)=0 \quad \text { for } q \geq 0
\end{array}\right.
$$

We are going to transform the problem (A.4), (A.5) into an equivalent problem to which Lemma A.1 can be applied.

In this goal, applying (3.48) and (3.62), for $i=0$, to the function $\mathcal{U}_{0}=\mathcal{W}_{p, 0}$, we see that the conditions (A.5) are equivalent to

$$
\begin{cases}\mathcal{W}_{p, 0}=j_{p, 0}+\sum_{q=0}^{+\infty} \ell_{q}^{1}\left(\mathcal{W}_{p, 0}\right) y_{q, 0} & \text { in } \widehat{\Omega}_{N}^{H} \\ \mathcal{W}_{p, 0}=\sum_{q=0}^{+\infty} L_{q}^{1}\left(\mathcal{W}_{p, 0}\right) d_{q, 0} & \text { in } \widehat{\Omega}_{N}^{S}\end{cases}
$$

Since, for all $q \geq 1$, the functions $y_{q, 0}$ (resp., $d_{q, 0}$ ) decay at infinity in $\widehat{\Omega}_{N}^{H}$ (resp., $\widehat{\Omega}_{N}^{S}$ ) and since $d_{0,0}=1$, one sees that (A.5) is also equivalent (see [12] for details) to

$$
\left\{\begin{array}{l}
\lim _{\rho \rightarrow+\infty}\left\{\mathcal{W}_{p, 0}(\rho, \theta)-j_{p, 0}(\rho, \theta)-\ell_{0}^{1}\left(\mathcal{W}_{p, 0}\right) y_{0,0}(\rho, \theta)\right\}=0 \\
\mathcal{W}_{p, 0} \text { is bounded in } \widehat{\Omega}_{N}^{S}
\end{array}\right.
$$

We next prove (4.23). For this, we integrate (A.4) over $\widehat{\Omega}_{N}^{I}$ and apply Green's formula to get

$$
0=\int_{\widehat{\Omega}_{N}^{I}} \Delta \mathcal{W}_{p, 0}=\int_{\partial \widehat{\Omega}_{N}^{I}} \frac{\partial \mathcal{W}_{p, 0}}{\partial n}=\int_{0}^{\pi} \frac{\partial \mathcal{W}_{p, 0}}{\partial \rho}(1, \theta) d \theta+\int_{-\frac{1}{2}}^{\frac{1}{2}} \frac{\partial \mathcal{W}_{p, 0}}{\partial X}(0, Y) d Y .
$$

Next, we use the modal expansions (A.6) and the fact that, for $q>0$, the functions $\cos q \theta$ (resp., $\left.\psi_{q}(Y)\right)$ have mean value 0 in $[0, \pi]$ (resp., $[-1 / 2,+1 / 2]$ ) to get

$\int_{0}^{\pi} \frac{\partial j_{0,0}}{\partial \rho}(1, \theta) d \theta+\ell_{0}^{1}\left(\mathcal{W}_{p, 0}\right) \int_{0}^{\pi} \frac{\partial y_{0,0}}{\partial \rho}(1, \theta) d \theta+L_{0}^{1}\left(\mathcal{W}_{p, 0}\right) \int_{0}^{1} \frac{\partial d_{0,0}}{\partial X}(1, Y) d Y=0$

which yields, since $j_{0,0}(\rho, \theta)=1, y_{0,0}(\rho, \theta)=1+\frac{2}{\pi} \log \rho$, and $d_{0,0}(X, Y)=1$,

$$
\ell_{0}^{1}\left(\mathcal{W}_{p, 0}\right)=0 .
$$

Hence, we have proven that (A.5) is equivalent to

$$
\left\{\begin{array}{l}
\lim _{\rho \rightarrow+\infty}\left\{\mathcal{W}_{p, 0}(\rho, \theta)-j_{p, 0}(\rho, \theta)\right\}=0 \\
\mathcal{W}_{p, 0} \text { is bounded in } \widehat{\Omega}_{N}^{S}
\end{array}\right.
$$

Finally, the existence and uniqueness of $\mathcal{W}_{p, 0}$ are ensured by Lemma A.1 with 


$$
f \equiv 0, \quad \mathcal{U}_{H}=j_{p, 0}, \quad \mathcal{U}_{S} \equiv 0
$$

Construction of $\mathcal{W}_{p, l}$ for $\boldsymbol{l} \geq \mathbf{1}$. We suppose that the $\mathcal{W}_{p, m}$ 's are defined for all $m<l$. Then, according to Lemma 4.3 , we look for $\mathcal{W}_{p, l}$ solution of

$$
\Delta \mathcal{W}_{p, l}=-4 \mathcal{W}_{p, l-2} \quad \text { in } \widehat{\Omega}_{N} \quad \text { and } \quad \frac{\partial \mathcal{W}_{p, l}}{\partial n}=0 \text { on } \partial \widehat{\Omega}_{N}
$$

and satisfying the conditions at infinity for any $m \leqslant l$ :

$$
\left\{\begin{array}{l}
\ell_{q}^{0}\left(\mathcal{W}_{p, m}\right)=1 \text { if } q=p \text { and } m=0 \quad \text { and } \quad \ell_{q}^{0}\left(\mathcal{W}_{p, m}\right)=0 \text { else } \\
L_{0}^{0}\left(\mathcal{W}_{p, m}\right)=L_{0}^{1}\left(\mathcal{W}_{p, m-1}\right) \quad \text { and } \quad L_{q}^{0}\left(\mathcal{W}_{p, m}\right)=0 \quad \text { for } q>0
\end{array}\right.
$$

First, we apply (3.48) and (3.62), for $i=l$, to the family $\left(\mathcal{W}_{p, i}\right)_{0 \leqslant i \leqslant l}$. Using (3.62) and the analytic expression of $c_{0, m}$ and $d_{0, m}(3.58)$, we get

$$
\begin{cases}\mathcal{W}_{p, l}=j_{p, l}+\sum_{q=0}^{+\infty} \sum_{m=0}^{l} \ell_{q}^{1}\left(\mathcal{W}_{p, l-m}\right) y_{q, m} & \text { in } \widehat{\Omega}_{N}^{H} \\ \mathcal{W}_{p, l}=\sum_{m=0}^{l} L_{0}^{1}\left(\mathcal{W}_{p, l-m}\right) \frac{(2 \mathbf{i} X)^{m}}{m !}+\sum_{q=1}^{+\infty} \sum_{m=0}^{l} L_{q}^{1}\left(\mathcal{W}_{p, l-m}\right) d_{q, m} & \text { in } \widehat{\Omega}_{N}^{S}\end{cases}
$$

Thanks to the Green-Riemann formula, one has

$$
\int_{\widehat{\Omega}_{N}^{I}} \Delta \mathcal{W}_{p, l}=\int_{\partial \widehat{\Omega}_{N}^{I}} \frac{\partial \mathcal{W}_{p, l}}{\partial n}=\int_{0}^{\pi} \frac{\partial \mathcal{W}_{p, l}}{\partial \rho}(1, \theta) d \theta+\int_{-\frac{1}{2}}^{\frac{1}{2}} \frac{\partial \mathcal{W}_{p, l}}{\partial X}(0, Y) d Y
$$

For $q>0$, the functions $\cos (q \theta)$ (resp., $\psi_{q}(Y)$ ) have mean value zero in $[0 ; \pi]$ (resp., $[-1 / 2 ; 1 / 2])$. Thus, (A.12) and (A.14) lead to

$$
\left\{\begin{aligned}
-4 \int_{\widehat{\Omega}_{N}^{I}} \mathcal{W}_{p, l-2}= & \int_{0}^{\pi} \frac{\partial \jmath_{p, l}}{\partial \rho}(1, \theta) d \theta+\sum_{m=0}^{l} \ell_{0}^{1}\left(\mathcal{W}_{p, l-m}\right) \int_{0}^{\pi} \frac{\partial y_{0, m}}{\partial \rho}(1, \theta) d \theta \\
& +2 \mathbf{i} L_{0}^{1}\left(\mathcal{W}_{p, l-1}\right)
\end{aligned}\right.
$$

Hence, since $\int_{0}^{\pi} \frac{\partial y_{0,0}}{\partial \rho}(1, \theta) d \theta=2$, we get the expression of $\ell_{0}^{1}\left(\mathcal{W}_{p, l}\right)$ :

$$
\left\{\begin{aligned}
\ell_{0}^{1}\left(\mathcal{W}_{p, l}\right)= & -\frac{1}{2} \int_{0}^{\pi} \frac{\partial \jmath_{p, l}}{\partial \rho}(1, \theta) d \theta-\frac{1}{2} \sum_{m=1}^{l} \ell_{0}^{1}\left(\mathcal{W}_{p, l-m}\right) \int_{0}^{\pi} \frac{\partial y_{0, m}}{\partial \rho}(1, \theta) d \theta \\
& -\mathbf{i} L_{0}^{1}\left(\mathcal{W}_{p, l-1}\right)-2 \int_{\widehat{\Omega}_{N}^{I}} \mathcal{W}_{p, l-2} \quad\left(\equiv \mu_{p, l}\right) .
\end{aligned}\right.
$$

For all $q>0, y_{q, 0}$ and $d_{q, 0}$ decay at infinity. Using a second time the modal expansions 
(A.14), one shows that conditions (A.13) are equivalent to (see [12] for details)

$$
\left\{\begin{array}{c}
\lim _{\rho \rightarrow+\infty} \sup _{\theta \in[0, \pi]}\left|\left\{\mathcal{W}_{p, l}-j_{p, l}-\mu_{p, l} y_{0,0}-\sum_{q=0}^{+\infty} \sum_{m=1}^{l}\left[\ell_{q}^{1}\left(\mathcal{W}_{p, l-m}\right) y_{q, m}\right]\right\}(\rho, \theta)\right|=0 \\
\mathcal{W}_{p, l}-\sum_{m=1}^{l} L_{0}^{1}\left(\mathcal{W}_{p, l-m}\right) \frac{(2 \mathbf{i} X)^{m}}{m !}-\sum_{q=1}^{+\infty} \sum_{m=1}^{l}\left[L_{q}^{1}\left(\mathcal{W}_{p, l-m}\right) d_{q, m}\right] \text { is bounded in } \widehat{\Omega}_{N}^{S} .
\end{array}\right.
$$

Let us set $f=4 \mathcal{W}_{p, l-2}$ and define $\mathcal{U}_{H}$ and $\mathcal{U}_{S}$ as

$$
\left\{\begin{array}{l}
\mathcal{U}_{H}=\jmath_{p, l}+\mu_{p, l} y_{0,0}+\sum_{q=0}^{+\infty} \sum_{m=1}^{l}\left[\ell_{q}^{1}\left(\mathcal{W}_{p, l-m}\right) y_{q, m}\right] \\
\mathcal{U}_{S}=\sum_{m=1}^{l} L_{0}^{1}\left(\mathcal{W}_{p, l-m}\right) \frac{(2 \mathbf{i} X)^{m}}{m !}+\sum_{q=1}^{+\infty} \sum_{m=1}^{l}\left[L_{q}^{1}\left(\mathcal{W}_{p, l-m}\right) d_{q, m}\right]
\end{array}\right.
$$

To apply Lemma A.1, it remains to check that $\mathcal{U}_{H}, \mathcal{U}_{S}$, and $f$ satisfy (A.1) and (A.3). To verify (A.1), we remark that (remember that $j_{q, 1}=y_{q, 1}=d_{q, 1}=0$ )

$$
\begin{aligned}
& \left\{\begin{aligned}
\Delta \mathcal{U}_{H} & =\Delta j_{p, l}+\mu_{p, l} \Delta y_{0,0}+\sum_{q=0}^{+\infty} \sum_{m=1}^{l}\left[\ell_{q}^{1}\left(\mathcal{W}_{p, l-m}\right) \Delta y_{q, m}\right] \\
& =\Delta j_{p, l}+\mu_{p, l} \Delta y_{0,0}+\sum_{q=0}^{+\infty} \sum_{m=2}^{l}\left[\ell_{q}^{1}\left(\mathcal{W}_{p, l-m}\right) \Delta y_{q, m}\right] \quad(\text { see }(3.39)) \\
& =\quad-4 \jmath_{p, l-2}-4 \sum_{q=1}^{+\infty} \sum_{m=0}^{l-2}\left[\ell_{q}^{1}\left(\mathcal{W}_{p, l-m-2}\right) \Delta y_{q, m}\right]=-4 \mathcal{W}_{p, l-2}=f
\end{aligned}\right. \\
& \quad(\operatorname{see}(\mathrm{A} .14)), \\
& \begin{aligned}
\Delta \mathcal{U}_{S}= & \sum_{m=1}^{l} L_{0}^{1}\left(\mathcal{W}_{p, l-m}\right) \Delta\left[\frac{(2 \mathbf{i} X)^{m}}{m !}\right]+\sum_{q=1}^{+\infty} \sum_{m=1}^{l}\left[L_{q}^{1}\left(\mathcal{W}_{p, l-m}\right) \Delta d_{q, m}\right] \\
= & \sum_{m=1}^{l} L_{0}^{1}\left(\mathcal{W}_{p, l-m}\right) \Delta\left[\frac{(2 \mathbf{i} X)^{m}}{m !}\right]+\sum_{q=1}^{+\infty} \sum_{m=2}^{l}\left[L_{q}^{1}\left(\mathcal{W}_{p, l-m}\right) \Delta d_{q, m}\right](\text { see }(3.5 \\
= & -4 \sum_{m=0}^{l-2} L_{0}^{1}\left(\mathcal{W}_{p, l-m-2}\right) \frac{(2 \mathbf{i} X)^{m}}{m !}-4 \sum_{q=1}^{+\infty} \sum_{m=0}^{l-2}\left[L_{q}^{1}\left(\mathcal{W}_{p, l-m-2}\right) d_{q, m}\right] \\
= & -4 \mathcal{W}_{p, l-2}=f \quad(\operatorname{see}(\mathrm{A} .14)) .
\end{aligned}
\end{aligned}
$$

To conclude, it is sufficient to check that

$$
I=\int_{0}^{\pi} \frac{\partial \mathcal{U}_{H}}{\partial \rho}(1, \theta) d \theta+\int_{-1 / 2}^{1 / 2} \frac{\partial \mathcal{U}_{S}}{\partial x}(0, Y) d Y+\int_{\widehat{\Omega}_{N}^{I}} f=0,
$$

which directly follows from (A.17) (we omit the details). 


\section{REFERENCES}

[1] P. Joly And S. ToRdeux, Asymptotic analysis of an approximate model for time harmonic waves in media with thin slots, M2AN Math. Model. Numer. Anal., 40 (2006), pp. 63-97.

[2] S. H. Sснот, Eighty years of Sommerfeld's radiation condition, Historia Math., 19 (1992), pp. $385-401$.

[3] M. Li, J. Nuebel, J. L. Drewniak, R. E. DuBroff, T. H. Hubing, and T. P. Van Doren, EMI from cavity modes of shielding enclosures - FDTD modeling and measurements, IEEE Trans. Electromagn. Compat., 42 (2000), pp. 29-38.

[4] V. A. Kondrat'Ev, Boundary value problems for elliptic equations in domains with conical or angular points, Trans. Moscow Math. Soc., 16 (1967), pp. 227-313.

5] M. Van Dyke, Perturbation Methods in Fluid Mechanics, Parabolic Press, Stanford, CA, 1975.

[6] M. Clausel, M. Duruflé, P. Joly, and S. Tordeux, A mathematical analysis of the resonance of the finite thin slots, Appl. Numer. Math., to appear.

[7] S. Tordeux, Un problème de Laplace non standard en milieu non borné, Tech. report 5799, INRIA, Le Chesnay Cedex, France, http://www.inria.fr (2006).

[8] P. HaRrington And D. Auckland, Electromagnetic transmission through narrow slots in thick conducting screens, IEEE Trans. Antennas and Propagation, 28 (1980), pp. 616-622.

[9] A. Taflove, K. Umashankar, B. Becker, F. Harfoush, and K. S. Yee, Detailed FDTD analysis of electromagnetic fields penetrating narrow slots and lapped joints in thick conducting screens, IEEE Trans. Antennas and Propagation, 36 (1988), pp. 247-257.

[10] A. Taflove, Computational Electrodynamics: The Finite-Difference Time-Domain Method, Artech House, Boston, 1995.

[11] G. VIAL, Analyse multiéchelle et conditions aux limites approchées pour un problème de couche mince dans un domaine à coin, Ph.D. Thesis, Université de Rennes I, Rennes Cedex, France, http://tel.ccsd.cnrs.fr (2003).

[12] S. Tordeux, Méthodes asymptotiques pour la propagation des ondes dans les milieux comportant des fentes, Ph.D. Thesis, Université de Versailles, Versailles Cedex, France, http://tel.ccsd.cnrs.fr (2004).

[13] V. G. Maz'ya, S. A. Nazarov, and B. A. Plamenevskit, Asymptotische Theorie elliptischer Randwertaufgaben in singulär gestörten Gebieten I, Math. Lehrbucher und Monogr. 82, Akademie-Verlag, Berlin, 1991.

[14] V. G. Maz'ya, S. A. Nazarov, and B. A. PlamenevskiI, Asymptotische Theorie elliptischer Randwertaufgaben in singulär gestörten Gebieten II, Math. Lehrbucher und Monogr. 83, Akademie-Verlag, Berlin, 1991.

[15] O. A. Oleinik, A. S. Shamaev, and G. A. Yosifian, Mathematical Problems in Elasticity and Homogenization, Stud. Math. Appl. 26, North-Holland, Amsterdam, 1992.

[16] D. G. Crighton, A. P. Dowling, J. E. Ffowks Williams, M. Heckl, and F. G. Leppington, Modern Methods in Analytical Acoustics: An Asymptotic Analysis, Lecture Notes 14, Springer-Verlag, London, 1992.

[17] A. M. IL'In, Matching of Asymptotic Expansions of Solutions of Boundary Value Problems, Transl. Math. Monogr. 102, AMS, Providence, RI, 1992.

[18] C. M. Butler And D. R. Wilton, General analysis of narrow strips and slots, IEEE Trans. Antennas and Propagation, 28 (1980), pp. 42-48.

[19] J. Gilbert And R. Holland, Implementation of the thin-slot formalism in the finite-difference EMP code THREDII, IEEE Trans. Nuc. Science, 28 (1981), pp. 4269-4274.

[20] G. A. Kriegsmann, The flanged waveguide antenna: Discrete reciprocity and conservation, Wave Motion, 29 (1999), pp. 81-95.

[21] P. McIver And A. D. Rawlins, Two-dimensional wave-scattering problems involving parallelwalled ducts, Quart. J. Mech. Appl. Math., 46 (1993), pp. 89-116.

[22] N. N. Lebedev, Special Functions and Their Applications, Prentice-Hall, Englewood Cliffs, NJ, 1965. 